Jens Kersten, Claudia Neu und Berthold Vogel

\title{
Die demografische Provokation der Infrastrukturen
}

\section{Infrastruktur, Daseinsvorsorge und Lebensverhältnisse}

Infrastrukturen dienen nicht nur unserer Versorgung, sondern sind Bedingungen gesellschaftlicher Integration. Deshalb manifestiert sich der demografisch bedingte Rückzug der Infrastruktur aus der Fläche nicht »nur « in Versorgungsproblemen. Vielmehr wirft er die Frage nach der sozialen, wirtschaftlichen und politischen Kohäsion der Bundesrepublik auf. Der demografische Wandel provoziert die vernetzte Gesellschaft, indem er mit der infrastrukturellen »Entnetzung « die Teilhabemöglichkeiten von Bürgerinnen und Bürgern beschränkt. Zugleich erodiert Öffentlichkeit, und Staatlichkeit verflüchtigt sich, wenn ganze Gemeinden und Regionen infrastrukturell »vom Netz « gehen. Die theoretische Reflexion dieser Entwicklung setzt bei dem sozialpolitischen Dreiklang von Infrastruktur, Daseinsvorsorge und Lebensverhältnissen an, der sich in den vergangenen 150 Jahren unter den Bedingungen wirtschaftlichen Wachstums funktional differenziert hat.

\subsection{Infrastrukturen}

»Infrastruktur « ist ein » verspäteter « Begriff. ${ }^{1}$ Er setzt sich erst in der zweiten Hälfte des 20. Jahrhunderts durch, um Verkehrs-, Versorgungs-, Gesundheits-, Kommunikations- und Bildungsnetze zu beschreiben, die sich seit Beginn der Industrialisierung verdichtet haben. ${ }^{2}$ Seit dem 19. Jahrhundert wird nicht nur die technische, sondern auch die soziale, politische, wirtschaftliche und rechtliche Bedeutung von raumgreifenden Verkehrsnetzen erkannt. Angesichts der sich zunehmend entfaltenden Verkehrs- und Versorgungsnetze verabschieden sich die alten Vorstellungen vom Raum als invariablem Territorialcontainer, eine Vorstellung, bei der Raum als räumlich-territorial eindeutig begrenzbare Einheit (Gemeinde, Stadt, Nation) erlebt wird. ${ }^{3}$ Es bildet sich ein neues, netzbasiertes Raumverständnis, das sich nicht mehr als ein »System der Raumüberwindung « ${ }^{4}$ begreift, sondern den sozialen Raum durch die zeitgenössisch neu aufkommenden Verkehrsmittel und telefonischen Kommunikationsmittel konstituiert sieht. So sprechen etwa Karl Marx und Friedrich Engels 1848 im Kommunistischen Manifest in Bezug auf die Eisenbahnen von » wachsenden Kommunikationsmitteln, die von der großen Industrie erzeugt werden

1 Vgl. hierzu und zum Folgenden Laak 1999, S. 280 ff.; Laak 2010, S. 9 ff.; Kersten 2008, S. $1 \mathrm{ff}$.

2 Vgl. Hermes 1998, S. 256 ff.

3 Vgl. Pries 1997; Graham, Marvin 2001, S. 73 ff.

4 Jhering 1894, S. 663.

Leviathan, 40. Jg., 4/2012, S. 563trit590 
und die Arbeiter der verschiedenen Lokalitäten miteinander in Verbindung setzen. « ${ }^{5}$ Hier fallen die zentralen Begriffe, die doch so sehr modern erscheinen: » Kommunikationsmittel « - »Industrie « - Verbindung von Lokalitäten « - also das, was wir heute als Netze und Netzwerke bezeichnen. Aber das 19. Jahrhundert bringt die besondere Funktion von technischen und sozialen Netzstrukturen noch nicht auf einen verallgemeinerungsfähigen Begriff. In Frankreich bezeichnet infrastructure noch 1875 und bis weit in die 1920er Jahre hinein den Unterbau von Eisenbahnkonstruktionen. ${ }^{6}$ Gleichwohl hat die liberale Verwaltungsrechtslehre im Deutschland des 19. Jahrhunderts sehr sensibel konstatiert, dass sich mit diesen Netzstrukturen auch die Staatsaufgaben exponentiell ausweiten: ${ }^{7}$ Nach Robert von Mohl und Rudolf von Gneist sollten die Bürger - in der Tradition der Revolution von 1848 den Aufbau der kommunalen Verkehrs- und Versorgungsnetze in Vereinen und Kommunen selbst gestalten. Auch Lorenz von Stein suchte mit seiner Unterscheidung zwischen "arbeitendem Staat « und »freier Verwaltung " nach Formen der Bürgerpartizipation für die Entfaltung von Verkehrs- und Versorgungsstrukturen. Und Otto Mayer rezipierte schließlich unter dem Eindruck der Infrastrukturstabilität um 1900 das französische Konzept des service publique in seiner Lehre von der »öffentlichen Anstalt ", die eine staatliche Verantwortung für die Netzstrukturen mit privater Aufgabenerfüllung kombiniert. Erst in den Wirtschaftskrisen und Weltkriegen der ersten Hälfte des 20. Jahrhunderts beginnt die Karriere des Infrastrukturbegriffs: ${ }^{8}$ Seit den 1940er Jahren finden sich immer häufiger Verwendungen des Begriffs »Infrastruktur " jenseits seines eisenbahntechnischen Ursprungs - wenngleich er in den 1950er Jahren noch als militärischer Strategiebegriff für ortsfeste Anlagen, die Voraussetzung für Mobilität sind, Anwendung findet. Erst ab den 1960er Jahren findet der Infrastrukturbegriff als technische und sozialpolitische Seite von Verkehrs-, Versorgungs-, Gesundheits-, Kommunikations- und Bildungsnetzen zu seiner heutigen allgemeinen Bedeutung.

\subsection{Daseinsvorsorge}

In Deutschland wird diese Entfaltung des Begriffs der »Infrastruktur " von der Entwicklung des sozialpolitischen Konzepts der »Daseinsvorsorge « durch Ernst Forsthoff begleitet. In seiner Monografie Die Verwaltung als Leistungsträger von 1938 erklärt Forsthoff - im nationalsozialistischen Zeitgeist befangen - die liberalen und partizipativen Infrastrukturkonzepte des 19. Jahrhunderts für politisch und rechtlich obsolet. Er bringt die staatliche Zentralisierung von Verkehrs-, Versorgungs-, Gesundheits-, Post-, Telefonie- und Bildungsstrukturen auf den Begriff der

5 Marx, Engels 1971 [1848], S. 535.

6 Laak 1999, S. $280 \mathrm{ff}$.

7 Vgl. hierzu und zum Folgenden Jellinghaus 2006, S. 143 ff., 159 ff., 167 ff., 259 ff. mit umfassenden Nachweisen.

8 Vgl. hierzu und zum Folgenden Laak 1999, S. 281 ff.; Laak 2010, S. 9; Jellinghaus 2006, S. 13 ff.; Frey 2005, S. 469 ff.; Wieland 2007, S. 377 ff. 
»Daseinsvorsorge «, ${ }^{9}$ den er bis in die 1970er Jahre zu einem umfassenden sozialpolitischen Legitimationskonzept des modernen Wohlfahrtsstaats weiterentwickeln wird. ${ }^{10}$ Dreh- und Angelpunkt von Forsthoffs Verwaltung als Leistungsträger ist die sozialpsychologische Grundierung der »Daseinsvorsorge" als Verwaltungsaufgabe: ${ }^{11}$ Die industrielle Revolution führt nach Forsthoff zu einem Wandel des sozialen Raumerlebnisses des Menschen. Der beherrschte Raum, der dem Einzelnen als Eigentum und Besitz zurechenbar ist, wird immer geringer. Der effektive Lebensraum, in dem sich das Leben tatsächlich vollzieht, wird aufgrund der Technisierung von Verkehr und Kommunikation immer größer. Der Mensch ist von seinen natürlichen Lebensressourcen abgeschnitten. Er kann seine Lebens- und Aneignungsbedürfnisse nicht mehr selbst befriedigen und muss über technische Apparate versorgt werden. Sozialpsychologisch führt dies dazu, dass der Einzelne seine Existenz als künstlich erlebt, sie deshalb als risikoreich reflektiert und nach Sicherheit in seiner Beziehung zur staatlichen Verwaltung strebt, die seine Daseinsvorsorge garantiert. Dieses Sicherheitsbedürfnis nach Daseinsvorsorge konstituiert die politische Gemeinschaft. So gibt der Einzelne lieber individuelle Freiheiten auf, als in einer Gesellschaft zu leben, in der er sein Daseinsrisiko selbst trägt. Er »tauscht « Freiheit in der Gesellschaft gegen Teilhabe an einer Gemeinschaft, die ihn vom Daseinsrisiko vollkommen entlastet. Insofern lautet denn auch der verfassungspolitische Programmsatz der Monografie: »Die Grundrechte gehören der Geschichte an. «12 An ihre Stelle tritt die Teilhabe. ${ }^{13}$ Diese ist jedoch nicht als subjektives Recht, sondern institutionell aus der "stets gemeinschaftsbezogen[nen] und pflichtgebunden[en] « »Rechtsstellung des Volksgenossen « zu fassen. ${ }^{14}$ Die staatliche Verwaltung übernimmt die "Daseinsverantwortung «15 für den Einzelnen. Dieser bindet sich im Gegenzug unauflöslich an die staatliche Verwaltung. Es entsteht eine Daseinsrisikogemeinschaft. Aus diesem sozialpsychologischen Tatbestand zieht Forsthoff den Schluss, dass solche Risikogemeinschaften politisch äußerst stabil sind. ${ }^{16}$ Zum einen delegitimiert der rationale und rechtliche Modus, dem die "Daseinsvorsorge " verpflichtet ist, alle Revolutionen außerhalb der »Legalität«: Ganz im Sinn der Rechtsgeltungslehre Max Webers muss nach Forsthoffs Auffassung jede politische Bewegung - einschließlich der NSDAP - in der Bevölkerung den psychologischen Eindruck erwecken, den Staat legal in die Hand bekommen zu haben und zu bedienen. Zum anderen ist die psychologische Verbindung des Einzelnen mit dieser staatlichen Daseinsrisikogemeinschaft so stark, dass das kollektive Risiko des Scheiterns

9 Vgl. Forsthoff 1938, S. 1 ff.

10 Vgl. hierzu und zum Folgenden Kersten 2005, S. 557 ff.; Schütte 2006, S. 81 ff.; Gegner 2007, S. 455 ff.; nun umfassend Meinel 2011, S. 154 ff.

11 Vgl. hierzu und zum Folgenden Forsthoff 1938, S. 3 ff., 9 f., 17 ff.

12 Ebd., S. 1; vgl. S. 43, 46.

13 Vgl. hierzu und zum Folgenden ebd., S. 15 ff., 19 ff., 38 ff., 46.

14 Vgl. ebd., S. 16.

15 Ebd., S. 6.

16 Vgl. hierzu und zum Folgenden ebd., S. 9, 18. 
dieser Gemeinschaft psychologisch ausgeblendet wird. Anderenfalls müsste - so Forsthoffs Begründung 1938 - angesichts eines »totalen Krieges « Panik ausbrechen, da sich ein solcher Krieg auch gegen die Einrichtungen der Daseinsvorsorge wende. ${ }^{17}$

Im Gegensatz zu dieser sozialpsychologischen Begründung und ihren verfassungspolitischen Folgerungen fällt das verwaltungsdogmatische Programm der Monografie wesentlich blasser aus. Die Probleme beginnen schon damit, dass Forsthoff angesichts der Weite seiner sozialpsychologischen Begründung "Daseinsvorsorge " nicht randscharf definieren kann. ${ }^{18}$ Grundsätzlich muss sie alle staatlichen Leistungen umfassen, die menschliche »Appropriationsbedürfnisse «19 befriedigen, einschließlich der sozialen Fürsorge. Allein im Hinblick auf die verwaltungsrechtlichen Folgerungen grenzt Forsthoff den Begriff auf zweiseitige Leistungsverhältnisse ein, auf die der Einzelne für seine Daseinsmöglichkeit angewiesen ist. ${ }^{20}$ In einer nicht abschließenden Kasuistik zählt er hierzu die »Versorgung mit Wasser, Gas, Elektrizität «, »die Bereitstellung der Verkehrsmittel jeder Art, die Post, Telephonie und Telegraphie, die hygienische Sicherung, die Vorsorge für Alter, Invalidität, Krankheit, Arbeitslosigkeit und vieles andere mehr", nicht jedoch Theater und "Lichtspiel «. Um die Lücke des liberalen Verwaltungsrechts für die Erfassung dieser Rechtsverhältnisse zu schließen, schlägt Forsthoff vor, Daseinsvorsorge als ein Rechtsinstitut zu verstehen. ${ }^{21}$ Dieses soll bestimmte Vorsorgeleistungen institutionell überformen, und dies unabhängig von der privaten oder öffentlichen Rechtsform, in der die Verwaltung oder ausnahmsweise die »freie " Wirtschaft diese Leistung erbringt. Doch in der dogmatischen Konturierung kommt Forsthoff nicht über eine Generalisierung der Grundsätze des Energiewirtschaftsgesetzes von 1935 hinaus, wobei er insbesondere die staatlichen Interventionsrechte, die allgemeine Anschluss- und Versorgungspflicht sowie die bindende Entscheidung über die Tarifgestaltung hervorhebt. ${ }^{22}$ In seinem Ausblick denkt Forsthoff über ein Rahmengesetz nach, das die Grundsätze der Daseinsvorsorge verallgemeinert, die sodann wieder in Sondergesetzen konkretisiert werden sollen. ${ }^{23}$ Auch mag sich Forsthoff grundsätzlich vorstellen, dass die »Daseinsverantwortung " für bestimmte Bereiche auf gemeinschaftsförmige Selbstverwaltungsordnungen unter der »subsidiären «»Oberaufsicht « des Staats übertragen werden kann. Doch hält er dies 1938 wegen der Einbindung der Daseinsvorsorge in die Kriegswirtschaft für ausgeschlossen. In dieser Zeit beginnt Forsthoff auch, sich von seiner nationalsozialistischen Überzeugung

17 Vgl. insbesondere ebd., S. 18; hierzu Kersten 2005, S. 553; darüber hinaus zu den »verborgenen Mächten der Panik « Meinel 2011, S. 163.

18 Vgl. hierzu und zum Folgenden Forsthoff 1938 S. 5 f., 47.

19 Ebd., S. 6.

20 Vgl. hierzu und zum Folgenden Forsthoff 1938, S. 7, 12, 42 ff., einschließlich der Zitate.

21 Vgl. hierzu und zum Folgenden ebd., S. 11 ff., 33 ff., 47 ff., einschließlich der Zitate.

22 Zum Gesetz über die Beförderung von Personen zu Lande vom 4. Dezember 1934 vgl. ferner Forsthoff 1938, S. 36 f.

23 Vgl. hierzu und zum Folgenden ebd., S. 48 ff.; hierzu auch Pauly 2001, S. 99. 
zu lösen und die Daseinsvorsorge nun nicht länger als Instrument zur totalen Kontrolle und Disziplinierung der Bevölkerung zu sehen. So betont er 1941 in seinem Vortrag Grenzen des Rechts, dass staatliche Daseinsvorsorge dem jeweiligen individuellen Bedarf angepasst sein muss. ${ }^{24}$

\subsection{Lebensverhältnisse}

Mit seiner sozialpolitischen Suggestionskraft hat das Konzept der »Daseinsvorsorge " die Staatsaufgabendiskurse der Nachkriegsjahrzehnte bis in die Politik der Europäischen Gemeinschaften geprägt. ${ }^{25}$ Doch selbst als der moderner anmutende Begriff der »Infrastruktur " zunehmend terminologisches Terrain gewinnt, wird dadurch die konzeptionelle Kopplung von sozialer Vorsorge und Teilhabe nicht aufgegeben, die auch die Grundpfeiler des Konzepts der Daseinsvorsorge bilden. Vielmehr verbindet sich ab den 1950er Jahren der Gedanke der »Daseinsvorsorge « bzw. der »Infrastrukturen « mit der politischen Vorstellung von der Herstellung gleichwertiger bzw. einheitlicher Lebensverhältnisse ${ }^{26}$ und erweitert auf diese Weise den sozialen Teilhabegedanken um eine föderale räumliche Dimension. Teilhabemöglichkeiten dienen nun nicht mehr allein dem sozialen Ausgleich zwischen Gesellschaftsschichten. Vielmehr sollen auch Menschen in benachteiligten Regionen über einen flächendeckenden Infrastrukturausbau an Errungenschaften der modernen, urbanen Wohlfahrts- und Konsumgesellschaft teilhaben können. Dabei wird bis heute die Konkretisierung der Leitvorstellung von der Gleichwertigkeit der Lebensverhältnisse durch die bundesrepublikanische Planungsphilosophie der späten 1950 er bis frühen 1970er Jahre geprägt. ${ }^{27}$ Diese Planungsphilosophie beschreibt keine faktische Realität, sondern setzt als Ausdruck des wirtschaftlichen Wachstums der Nachkriegsjahrzehnte auf eine Politik der Konvergenz und damit des Ausgleichs von räumlichen Disparitäten zwischen den Bundesländern, aber auch zwischen Stadt und Land. Ganz dem wohlfahrtsstaatlichen Partizipationsversprechen dieser Jahrzehnte folgend, bezieht sich dieser Ausgleich auf alle Lebensbereiche: Arbeit, Bildung, Einkaufen, Erholung, Freizeit, Kultur, Gesundheit, Sozialleistungen, Telekommunikation, Verkehr und Wohnen. ${ }^{28}$ Maßstab für die Bestimmung der durch das Leitbild geforderten Gleichwertigkeit ist der "gesellschaftlich akzeptierte Standard «, wobei Ausgleich in der bis dato gängigen Interpretation stets als eine Angleichung nach »oben «- also an ein hohes wohlfahrtsstaatliches Niveau - verstanden wird. ${ }^{29}$

24 Vgl. Forsthoff 1941, S. 20.

25 Vgl. Kersten 2005, S. 565 ff.

26 Vgl. Artikel 72 Absatz 2, Artikel 106 Absatz 3 Satz 4 Nummer 2 Grundgesetz (GG).

27 Vgl. hierzu und zum Folgenden Zimmermann 2005, S. 13; Laak 2010, S. 14; Kersten 2006 a, S. 245 ff.; Reichel 2009, S. 7 ff., 31 ff.

28 Vgl. Runkel 2005, K $\mathbb{1}$, Rn. 85 f.

29 Vgl. BMVBW, BBR 2005, S. 22.

Leviathan, 40. Jg., 4/2012 


\section{Demografischer Wandel und infrastrukturelle Desintegration}

Seit den 1970er Jahren verändern Geburtenrückgang, Wanderungsbewegungen und Langlebigkeit die Größe und Zusammensetzung der Bevölkerung. Schrumpfung und Alterung der Gesellschaft stellen zeitlich verzögert auch die Frage nach dem Zusammenhang von infrastruktureller Daseinsvorsorge und Wohlfahrtsstaat. ${ }^{30} \mathrm{Als}$ scheinbar »weiches " politisches Thema wurde und wird die demografische Entwicklung in der kollektiven Aufmerksamkeit durch scheinbar "harte " politische Agenden wie beispielsweise die Wiedervereinigung, den Klimawandel oder die Finanz- und Schuldenkrise verdrängt. Doch zum einen verfügen auch diese »harten « politischen Themen alle über demografische Implikationen; ${ }^{31}$ zum anderen zeitigt die demografische Entwicklung ebenfalls infrastrukturelle Folgen: Während der Strukturwandel der Industriegesellschaft unter den Bedingungen der Europäisierung und Globalisierung zu vertikalen sozialen Wohlstandskonflikten führt, ${ }^{32}$ stellt der demografische Wandel die Politik vor die »räumliche« soziale Frage der infrastrukturellen Versorgung ganzer Gemeinden und Regionen. Diese Entwicklung ist sicherlich nicht allein, aber eben auch dem demografischen Wandel geschuldet: Die lokalen und regionalen Schwächen von Arbeitsmärkten und Wirtschaftsstrukturen fördern ebenfalls das soziale und wirtschaftliche Gefälle in der bundesrepublikanischen Raumstruktur. Doch diese Strukturdefizite werden durch die demografisch induzierte Infrastrukturkrise noch weiter verstärkt und drohen ganze Gemeinden und Regionen in eine soziale und wirtschaftliche Abwärtsspirale zu reißen. Sie provozieren auf diese Weise die dreidimensionale Verbindung von Infrastrukturen, Daseinsvorsorge und Gleichwertigkeitspostulat, welche im Grundgesetz festgeschrieben ist und das historisch gewachsene Selbstverständnis des Wohlfahrtsstaats bis heute prägt.

\subsection{Ausweitung der Entleerungsgebiete und Verschärfung des Infrastrukturgefälles}

Was zunächst nur als die Transformationslast ostdeutscher Kommunen infolge von De-Industrialisierung und Abwanderung nach dem Abbau der ostdeutschen Betriebe erschien, hat sich zu einem flächendeckenden Phänomen entwickelt, das vor allem das Dreieck Düsseldorf-Stralsund-Dresden sowie die Küstenregionen umfasst. Diese demografischen Entleerungsgebiete werden von den wachstumsstarken Metropolregionen kontrastiert, die sich von Hamburg über Bremen, RheinRuhr, Rhein-Main, Rhein-Neckar und Stuttgart bis nach München spannen und in Ostdeutschland allein durch den Wachstumspunkt Berlin ergänzt werden. ${ }^{33}{ } \mathrm{C}$ mit

30 Vgl. Kersten et al. 2012 b, insbesondere Kapitel III.

31 Vgl. beispielhaft zum Verhältnis von Demografie und Wiedervereinigung Neu 2009, S. 81 ff.; zum Verhältnis von Demografie und Klimawandel Battis et al. 2010, S. 256; zum Verhältnis von Demografie und Schuldenkrise Pünder 2008, S. 946 ff; zu Demografie und Infrastruktur auch Kersten et al. 2012 a, S. 42-59.

32 Vgl. Dahrendorf 2004 [1995], S. 117; Castel 2005, S. 13 ff., 54 ff.; Vogel 2007, S. 71 ff.; Vogel 2009.

33 Vgl. hierzu und zum Folgenden BMVBW, BBR 2005, S. 7 ff. 
Punkt « lautet deshalb die »neue« wirtschaftsgeografische Formel für die Bundesrepublik. Diese Gleichzeitigkeit im Nebeneinander von Wachstums- und Schrumpfungsregionen verstärkt jedoch nur die Wahrnehmung der infrastrukturellen Brüche und Gefälle, mit denen sich die Frage nach der sozialen, wirtschaftlichen und politischen Kohäsion der Bundesrepublik insgesamt stellt.

\subsection{Soziale Desintegrationseffekte und ordnungspolitische Dysfunktionalitäten}

Zunächst galt dieser spatial turn der sozialen Frage als ein Problem peripherer ländlicher Räume in Ostdeutschland, in denen zwei demografische Phänomene zusammentrafen: ${ }^{34}$ Einerseits schrumpften aufgrund der niedrigen Geburtenraten sowie von Wanderungsverlusten in den Nachwendejahren zunächst Kindergartengruppen und sodann Schulklassen; andererseits stieg mit der erhöhten Lebenserwartung der soziale und medizinische Betreuungs- und Pflegebedarf älterer Bürgerinnen und Bürger. Gleichzeitiges Schrumpfen und Altern der Bevölkerung löst eine Infrastrukturkrise aus, deren technische, finanzielle, administrative, soziale und politische Dimensionen sich gegenseitig verstärken. Um dies zu verdeutlichen: Weniger Nutzer führen in technischer Hinsicht zur Unterauslastung der Wasser- und Abwasserleitungen, die nicht mehr hinreichend gespült werden. Der Nachfragerückgang und das reduzierte Steueraufkommen stellen in etlichen Regionen die finanzielle Tragfähigkeit von Bildungs-, Gesundheits-, Telekommunikations- und Verkehrsinfrastrukturen in Frage. Das Zurückfahren der staatlichen Sicherheits- und Verwaltungsinfrastruktur ist die Folge. Abwanderung und Geburtenrückgang lassen auch die örtlichen und regionalen Vereins- und Parteieninfrastrukturen zunächst »überaltern « und dann mitunter auch langsam vollkommen verlöschen. Doch es greift zu kurz, in der Erosion dieser vernetzten Gesellschaft, die sich durch solche starken infrastrukturellen Verflechtungen auszeichnet, allein eine Gefahr für die soziale, territoriale und politische Binnenintegration einer Gemeinde oder einer Region zu sehen. Vielmehr zeitigt sie auch Wirkung für ihre Integration in die bundesrepublikanische und - weiter gefasst - die europäische Gesellschaft. Denn wie eingangs gezeigt, hat die Evolution der Infrastrukturen seit dem 19. Jahrhundert zu einem Wandel des sozialen Raumverständnisses geführt: Der soziale Raum, in dem sich die gesellschaftliche Integration vollzieht, wird durch infrastrukturierende Technikverbindungen und Telekommunikationsbeziehungen konstituiert. ${ }^{35}$

Doch schon der Wegfall einzelner Infrastrukturbausteine kann sozialpolitische Desintegrationseffekte nach sich ziehen: Wenn beispielsweise die Zahl der Schulkinder zurückgeht, sinkt die quantitative Nachfrage nach öffentlichen Nahverkehrsverbindungen. Dies beschränkt die Möglichkeiten älterer Bürgerinnen und Bürger, einen Arzt zu erreichen. Diese Effekte verstärken sich, indem die in Schrumpfungsgebieten ohnehin zurückgehende ärztliche Niederlassungsdichte für viele die Wege zur medizinischen Versorgung verlängern. Damit werden Schrump-

34 Vgl. hierzu und zum Folgenden Neu 2006, S. 8 ff.; zur gestiegenen Lebenserwartung BiB 2011.

35 Siehe auch Barlösius et al. 2011, S. $158 \mathrm{f}$.

Leviathan, 40. Jg., 4/2012 
fungsregionen für junge wie ältere Menschen unattraktiv: Junge Paare mit Kinderwunsch oder Kindern ziehen aufgrund der schlechten Kinder- und Schulinfrastruktur nicht zu bzw. wandern ab. Doch in peripherisierten Regionen drohen für die Bevölkerung nicht nur Versorgungsengpässe. Auf sie kommt zugleich eine erhöhte Abgabenlast zu, wenn die Kosten beispielsweise für längere Wege oder für Wasser und Abwasser proportional mit der Abwanderung potenzieller Infrastrukturnutzer steigen. Folgen zeigen sich auch auf dem Immobilienmarkt, wenn Grundstücksinfrastrukturen in eine negative Kostenspirale geraten und die sinkende Nachfrage zugleich den wirtschaftlichen Wert immobilen Eigentums verfallen lässt. Mit diesem ökonomisch-demografisch induzierten Verfall der Immobilienwerte verliert für viele Bürgerinnen und Bürger ihr einziger "großer " Vermögensgegenstand seinen Wert, auf den sie vor allem auch hinsichtlich ihrer Alterssicherung gesetzt haben. ${ }^{36}$ Damit ist nicht gesagt, dass der Schrumpfungsprozess nicht auch - im wahrsten Sinn des Wortes - »Raum für Neues « schaffen kann: für »Raumpioniere «, ${ }^{37}$ die ihren sozialen Eigensinn erproben wollen, und insbesondere für die "Rückeroberung « von Kulturlandschaften durch die Natur. Doch diese "Chancen des Schrumpfens" beantworten nicht die Infrastrukturfrage, die durch die ökonomischen und demografischen Entwicklungen aufgeworfen wird und die die sozialstrukturelle Mitte der schrumpfenden Gesellschaft sozial, wirtschaftlich und politisch verunsichert.

Auch die Politik versucht, spät und zögerlich, sich den Problemen der flächendeckenden Aufrechterhaltung der Daseinsvorsorgeleistungen zu stellen. So werden etwa vom Bundesministerium des Innern (BMI) in seinem Handlungskonzept »Daseinsvorsorge im demografischen Wandel zukunftsfähig gestalten «, das im August 2011 erschien, Erfahrungen der ostdeutschen Bundesländer aufgegriffen und innovative Lösungen im Umgang mit Schrumpfung und Alterung vorgestellt. In diesem Konzept werden als Lösungsvorschläge ebenso Effizienzsteigerungen durch Erneuerung der Infrastruktur genannt wie auch die Notwendigkeit, Bürgern neue, flexible, auf die demografische Entwicklung reagierende Handlungsspielräume zu eröffnen. Die im März 2012 vorgelegte »Demographiestrategie« der Bundesregierung bleibt hingegen deutlich hinter den Erwartungen für eine zukunftsweisende demografische Leitlinie zurück. ${ }^{38}$ Auch das Bundesministerium für Ernährung, Landwirtschaft und Verbraucherschutz (BMELV) versucht spätestens seit Beginn des neuen Jahrtausends - konzentriert auf das Engagement von Bürgerinnen und Bürgern sowie den Einsatz von Regionalbudgets -, ländliche Räume $\mathrm{zu}$ »aktivieren «. Das von Renate Künast ins Leben gerufene Programm »Regionen aktiv « (2002-2007) wurde durch das neue Programm »LandZukunft « (2012-2014) abgelöst, das fünf Modellstandorte und fünf Sonderprojekte mit umfangreichen Finanzmitteln eigenverantwortlich ausstattet. ${ }^{39}$

36 Vgl. Davy 2007 a, S. 58 ff.; Davy 2007 b, S. 145 f.

37 Vgl. Lange, Matthies 2005, S. 374-383.

38 Vgl. Beauftragter der Bundesregierung für die Neuen Länder 2011; BMI 2012.

39 Vgl. BMELV 2008; BMELV 2012. 


\subsection{Verlust an Öffentlichkeit und Diffusion von Staatlichkeit}

Die demografische Entwicklung führt jedoch nicht nur zu infrastrukturellen Einbußen an wohlfahrtsstaatlicher Versorgungssicherheit, sondern auch zu einem Verlust an pluralistischer Öffentlichkeit und zu einer Diffusion von demokratischer Staatlichkeit: Die Schließung von Schulen, Verwaltungen, Rathäusern und Polizeidienststellen sowie die Einschränkung des öffentlichen Personennahverkehrs reduzieren den öffentlichen und sicheren Raum, in dem sich - im Zusammenspiel mit einem lokalen Vereins- und Parteienleben - eine pluralistische Öffentlichkeit diskursiv entfalten kann und in dem über das staatliche Gewaltmonopol die körperliche Sicherheit aller Bürgerinnen und Bürgern gewährleistet ist. Wenn darüber hinaus Arztpraxen nicht fortgeführt, Kirchen aufgegeben und Geschäfte geschlossen werden, verliert eine Gemeinde gleichzeitig Orte mit öffentlichem Publikumsverkehr. Unter den Bedingungen des demografischen Wandels kommen der Zivilgesellschaft als »Sphäre der zum Publikum versammelten Privatleute « 40 mithin nicht nur die öffentlichen und sicheren Orte, sondern zugleich das engagierte Publikum abhanden: Mit den sich aus der Fläche zurückziehenden Ärzten, Selbstständigen und Beamten fehlen einer Gemeinde und Region aktive Menschen, von deren Engagement das Kultur-, Vereins- und Parteienleben einer bürgerlichen Öffentlichkeit vielfach abhängig ist.

Das Alarmierende an dieser Zunahme sozialer Verunsicherung in Verbindung mit dem gleichzeitigen Verlust pluralistischer Öffentlichkeit und der Diffusion demokratischer Staatlichkeit ist jedoch, dass dadurch ein soziales und politisches Vakuum entstehen kann. Denn es besteht die Gefahr, dass die sozialpolitische »Leere ", die der Kollaps von Öffentlichkeit und der Rückzug von Staatlichkeit hinterlassen, von autoritären und oft extremistischen Kräften ausgefüllt wird, die nun ihrerseits gesellschaftliche Ordnungsfunktionen übernehmen. So kann ein politischer Raum für die Herrschaft des »Oberförsters « entstehen, dem eine »Wolke von Furcht « vorausgeht: ${ }^{41}$ Wohin - so lautet die wohl prekärste und zugleich ursprünglichste Frage der Politik - flüchtet sich eine Bürgerin oder ein Bürger in diesem parakonstitutionellen Raum, wenn sie oder er körperlich bedroht wird?

\subsection{Auszehrung lokaler Mittelschichten und der Substanzverlust öffentlicher Leistungen}

Infrastruktur und Daseinsvorsorge sind daher nicht nur abstrakte Prinzipien oder staatlicherseits zu gewährleistende Organisationsformen des Sozialen. Sie gewinnen Gestalt und Leben durch ihre Trägergruppen, durch diejenigen, die mit ihrer Tätigkeit und Profession öffentliche Leistungen ermöglichen, gewähren und verwalten. Während sich im bürgerschaftlichen Engagement - in Vereinen, Kirchengemeinden, Bürgerinitiativen - in der Regel partikulare Interessen spiegeln, sind die Beschäftigten der öffentlichen Dienstleistungen und Güterproduktion - die Verwaltungsangestellten, die Erzieher, die Busfahrer oder die Polizisten - mit der Verpflichtung auf

40 Habermas 1990 [1962], S. 42.

41 Vgl. Jünger 1978 [1938], S. 265 ff., bes. S. 269 (Zitat).

Leviathan, 40. Jg., 4/2012 
die Angelegenheiten des Gemeinwohls beauftragt. Vor allem die kommunale Verwaltung und ihr Personal kommen an dieser Stelle in den Blick. Doch sie sind nicht nur eine spezifische Professionsgruppe in der Vielfalt der Arbeitswelt, sie repräsentieren als Bürger auch die lokale Mittelschicht. Der öffentliche Dienst in seinen unterschiedlichen Abstufungen und Karrierepfaden, in seiner Beschäftigungssicherheit und seiner stark regulierten Arbeitswirklichkeit bildet das "juste milieu ", das auch in seiner Mentalität und Orientierung für lokale Stabilität und Integration sorgt. ${ }^{42}$ Allerdings sehen sich gerade die Akteure in der öffentlichen Verwaltung seit einigen Jahren mit starken Veränderungen ihrer Arbeitsbedingungen konfrontiert insbesondere in den Regionen, in denen die selbstverständliche Gegenwart und Zukunft öffentlicher Daseinsvorsorge und Infrastrukturleistung in Frage steht. Was heißt das für die Leistungsfähigkeit öffentlicher Dienste? Welche Konsequenzen hat das für die lokalen Sozialstrukturen? Die öffentlichen Güter und Dienste verlieren im Prozess demografischer Schrumpfung an Substanz. Sie fallen knappen Kassen zum Opfer, die Arbeitsbedingungen in den öffentlichen Einrichtungen verschlechtern sich und werden auch für die junge Generation als Arbeitsort und Berufsfeld immer unattraktiver. Nicht nur dadurch, dass viele Länder Stellenstopps eingeführt haben, geht vom Angebot öffentlicher Dienstleistungen immer weniger soziale, kulturelle und berufliche Bindekraft aus. Auch die Beschäftigungsbedingungen und beruflichen Entwicklungsmöglichkeiten im öffentlichen Sektor haben in den vergangenen Jahren deutlich an Attraktivität verloren. Im Personalbestand spiegeln sich diese Entwicklungen - in den Schulen und Behörden, aber auch in der Gesundheitsversorgung »überaltern" die Beschäftigten. Der Landarzt, der - längst im Pensionsalter - keine Nachfolge für seine Praxis findet, ist bereits legendär und Gegenstand politischer Reformbemühungen. Diese typischen Folgen des Schrumpfungsprozesses - das Verschwinden der Landarztpraxis, die drastische Reduzierung des kommunalen Verwaltungspersonals oder der Wegfall der örtlichen Polizeidienststelle - haben also nicht nur organisatorische Konsequenzen, sondern sie reißen zugleich empfindliche Lücken in das lokale und regionale Sozialgefüge. Die Mitte der Gesellschaft schwindet und »überaltert «, wenn der Generationenwechsel Einsparzwängen zum Opfer fällt. Der Verlust öffentlicher Einrichtungen und die Diffusion von Staatlichkeit verändern die soziale Lebenswirklichkeit. Typische Berufe der Mittelschicht, die Sicherheit und Auskommen bieten, die gerade deswegen auch Raum für außerberufliches Engagement lassen, verlieren an Gewicht. Der Substanzverlust von Infrastruktur und Daseinsvorsorge zehrt die Mitte lokaler Gesellschaften aus. Die wichtigsten Trägergruppen bürgerschaftlichen Engagements stehen unter Druck. Auf diese Weise wird deutlich, dass Infrastrukturen in soziologischer Hinsicht nicht nur Versorgungsleistungen repräsentieren, deren Fehlen oder Vorhandensein technische, räumliche oder verwaltungsbezogene Folgen nach sich ziehen. Der Ausbau und die Sicherung der Infrastruktur produziert ebenso eine spezifische Sozialstruktur. Mehr noch: Infrastrukturen stabilisieren soziale Strukturen. Der Infrastrukturabbau lässt somit auch die Tragfähigkeit und Kohäsionskraft sozialer Strukturen schwinden. 


\section{Infrastruktureller Rückzug aus staatlicher und bürgerlicher Perspektive}

Obwohl die demografischen Daten und Fakten sowie die Bevölkerungsprognosen bis 2060 "auf dem Tisch « liegen, fällt deren politische Wahrnehmung sowohl aus der Perspektive des Staats als auch der Bürgerinnen und Bürger schwer. Dies zeigt, wie nachhaltig der historisch tradierte Dreiklang aus Infrastrukturen, Daseinsvorsorge und Gleichwertigkeitsprinzip das kollektive Selbstverständnis der sozialräumlichen Struktur der Bundesrepublik bis heute prägt. Es herrscht Ratlosigkeit und banges Hoffen, »es werde schon nicht so schlimm kommen «. Deshalb wird auch allzu oft nur halbherzig nach neuen Partnern im Umgang mit den Folgen des demografischen Wandels gesucht, und die politischen Strategien - Ignorieren oder Neugestalten - reflektieren in den wenigsten Fällen die Vorstellungen oder den zu erwartenden Bedarf der Bürgerinnen und Bürger im Hinblick auf die zukünftige Gestaltung der Daseinsvorsorge.

\subsection{Staatliche Perspektive}

Der Staat hat vor allem zwei Formen entwickelt, um mit den Konsequenzen der demografisch induzierten Infrastrukturkrise umzugehen. Zum einen wird der demografische Wandel in einigen Gemeinden und Regionen defensiv ignoriert: Es kommt zu einer »kalten Sanierung « der Infrastrukturen, die schlicht »absterben ${ }^{43}{ }^{43}$ Nicht selten drohen Versorgungsengpässe für die Bevölkerung. Mitteleinsparungen, die durch die Zusammenlegung staatlicher Institutionen etwa im schulischen Bereich entstehen, werden beispielsweise durch erhöhte Transportkosten für die Schülerinnen und Schüler wieder "aufgefressen " oder einseitig zu Lasten der Kunden verlagert. ${ }^{44}$ Teilweise wird versucht, an »allen Fronten « harte Einschnitte und klare Prioritätensetzung mit der Folge zu vermeiden, dass schließlich in allen Bereichen die Mittel für eine nachhaltige Infrastrukturpolitik fehlen. Zum anderen versuchen Gemeinden und Regionen - unter den Bedingungen einer angespannten öffentlichen Haushaltslage -, die demografische Herausforderung offen anzugehen: Durch den Erhalt des Kindergartens, der Ausweisung von Bauland für junge Familien oder der Neugründung von Mehrgenerationenhäusern sollen Abwanderungen gestoppt und Neubürger gewonnen werden. So bewegt sich die Anpassung oder vermeintliche infrastrukturelle Neugestaltung weiterhin in den herkömmlichen Bahnen einer Politik, die auf Zukunftsgestaltung durch Wachstumsangebote setzt. Diese politischen Hoffnungen und Absichten bleiben in konventionellen und bewährten Bahnen. Wer bestimmten Gruppen der Bevölkerung »mehr « bietet, der setzt sich durch. Demgegenüber werden die komplexen gesellschaftlichen Aushandlungsprozesse als Voraussetzungen von Infrastrukturen ebenso wenig thematisiert wie deren Funktionen, Aufgaben und Ziele. Doch ohne eine solche Auseinandersetzung und Funktionsbe-

43 Vgl. darüber hinausgehend zur Frage von Umzugs- und Wegzugsprämien Canzler, Knie 2009, S. 109; Becker et al. 2001, S. 13.

44 Vgl. BBR 2007, S. 8 f.

Leviathan, 40. Jg., 4/2012 
stimmung lässt sich die infrastrukturelle Daseinsvorsorge unter den Bedingungen des demografischen Wandels nicht nachhaltig gestalten.

\subsection{Bürgerschaftliche Perspektive}

Dieser schleichende infrastrukturelle Rückzug des Staats aus einigen Regionen wird begleitet von einem verstärkten Ruf nach einer aktiven Bürgerschaft, die von politischer Seite aufgefordert wird, ihre Daseinsvorsorge nun mutig in die eigenen Hände zu nehmen: ${ }^{45}$ Bürgerbusse, Nachbarschaftshilfe, Dorfläden sind die Stichworte für diese neue bürgerliche Selbstständigkeit, die, ergänzt durch Leistungsangebote freier Träger und privater Anbieter, die infrastrukturellen Lücken schließen sollen. Um jedoch die bürgerschaftliche Perspektive auf die demografische Provokation der Infrastrukturen näher erschließen zu können, gilt es zwischen der Verantwortungs- und der Bedarfsstruktur der Daseinsvorsorge zu differenzieren.

\section{Verantwortungsstruktur der Daseinsvorsorge}

Die empirische Untersuchung der Verantwortungsstruktur veranschaulicht, wer nach Auffassung der Bürgerinnen und Bürger für die Erbringung der Daseinsvorsorgeleistungen verantwortlich sein soll. Wird zunächst nach den rein technischen Infrastrukturen gefragt, so bevorzugen die Bürgerinnen und Bürger die Daseinsvorsorgeleistungen nach wie vor aus »starken « kommunalen Händen. So wünschten sich 2008 in einer repräsentativen Haushaltskundenumfrage 58 Prozent der befragten Personen eine Stromversorgung durch die Stadtwerke (Abbildung 1) und lehnten zugleich 75 Prozent eine Wasserversorgung durch private Anbieter ab (Abbildung 2). ${ }^{46}$

In die gleiche Richtung weisen die Ergebnisse einer Gemeindestudie aus Mecklenburg-Vorpommern. ${ }^{47}$ Die befragten Bürgerinnen und Bürger der Gemeinde Galenbeck schreiben ebenfalls »staatlichen Stellen «- also Bund, Ländern und Kommunen - im Vergleich mit Unternehmen, freien/kirchlichen Trägern und eigeninitiativen Bürgerinnen und Bürgern die größere Kompetenz bei der Infrastrukturbereitstellung zu (Abbildung 3).

Auf einzelne Bereiche der Daseinsvorsorge hin befragt, stellt sich das Bild einer Verantwortungsteilung zwischen Staat, Unternehmen, freien/kirchlichen Trägern und eigeninitiativen Bürgern für die Daseinsvorsorge jedoch bereits differenzierter dar (Tabelle 1).

45 Vgl. differenzierend Albrecht 2009, S. 206 ff.; grundsätzlich BMI 2012, S. 42.

46 Vgl. dimap 2008.

$47 \mathrm{Vgl}$. Neu et al. 2007. 


\section{Abbildung 1: Bevorzugte Stromanbieter}

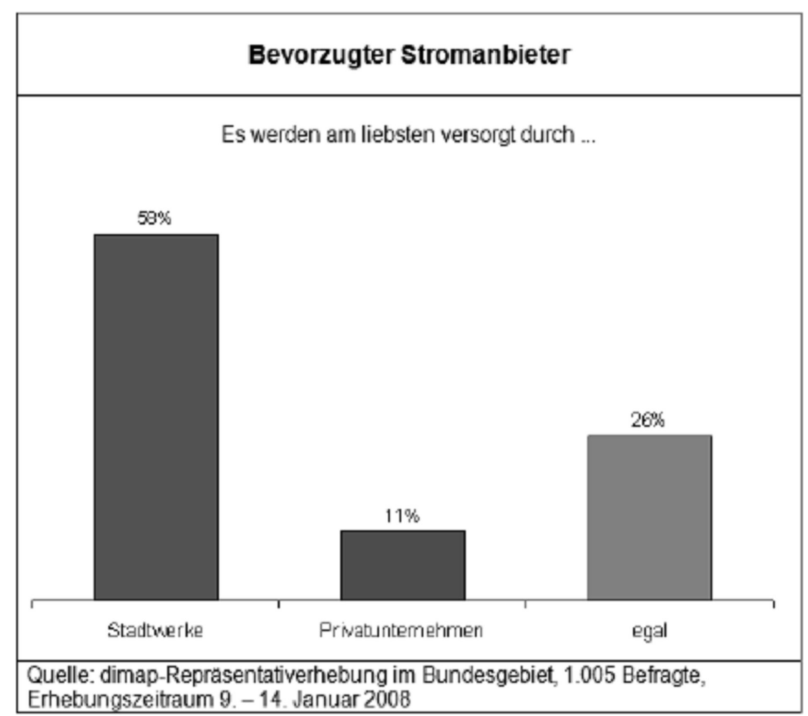

Quelle: dimap 2008.

\section{Abbildung 2: Bevorzugte Trinkwasserversorgung}

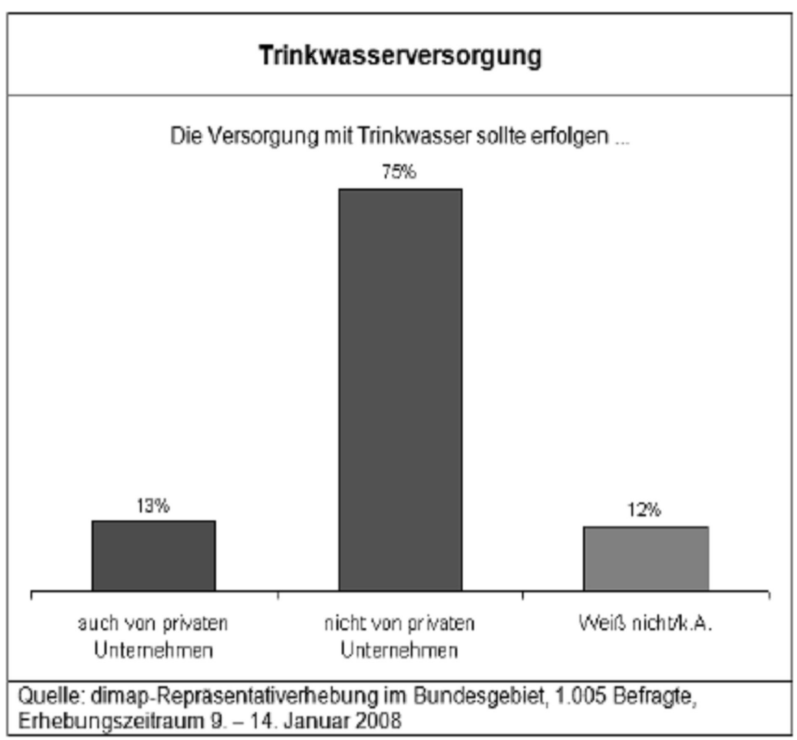

Quelle: dimap 2008. 
Abbildung 3: Kompetenzzuordnung für Infrastrukturleistungen gesamt (in Prozent)

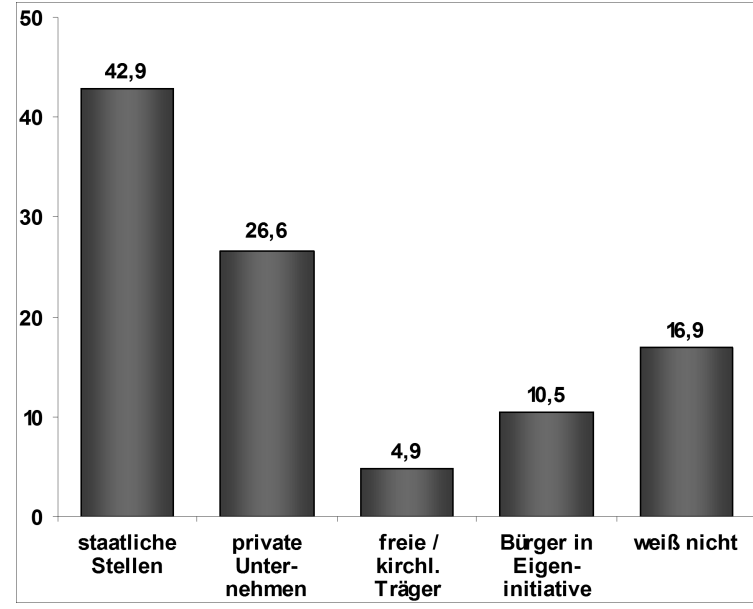

Quelle: Neu et al. 2007; eigene Erhebung.

Tabelle 1: Zuständigkeit für Infrastruktur

\begin{tabular}{|l|c|c|c|c|c|}
\hline & $\begin{array}{c}\text { Staatliche } \\
\text { Stellen }\end{array}$ & $\begin{array}{c}\text { Private } \\
\text { Unterneh- } \\
\text { mer }\end{array}$ & $\begin{array}{c}\text { Freie/ } \\
\text { kirchl. } \\
\text { Träger }\end{array}$ & $\begin{array}{c}\text { Bürger in } \\
\text { Eigeninitia- } \\
\text { tive }\end{array}$ & $\begin{array}{c}\text { Weiß } \\
\text { nicht }\end{array}$ \\
\hline Straßenbau & $77 \%$ & $13 \%$ & $0 \%$ & $1 \%$ & $10 \%$ \\
\hline Öffentliche Sicherheit & $82 \%$ & $9 \%$ & $0 \%$ & $3 \%$ & $8 \%$ \\
\hline Schulen & $74 \%$ & $6 \%$ & $6 \%$ & $2 \%$ & $15 \%$ \\
\hline Wasser/Abwasserversorgung & $49 \%$ & $36 \%$ & $3 \%$ & $4 \%$ & $10 \%$ \\
\hline ÖPNV & $40 \%$ & $38 \%$ & $2 \%$ & $3 \%$ & $18 \%$ \\
\hline Feuerwehr & $43 \%$ & $3 \%$ & $5 \%$ & $41 \%$ & $10 \%$ \\
\hline Schwimmbäder & $35 \%$ & $30 \%$ & $2 \%$ & $4 \%$ & $28 \%$ \\
\hline Kindergärten & $37 \%$ & $30 \%$ & $7 \%$ & $5 \%$ & $16 \%$ \\
\hline Ärztliche Versorgung & $34 \%$ & $51 \%$ & $1 \%$ & $0 \%$ & $14 \%$ \\
\hline Seniorenbetreuung & $35 \%$ & $24 \%$ & $19 \%$ & $9 \%$ & $17 \%$ \\
\hline Bankfilialen & $41 \%$ & $35 \%$ & $0 \%$ & $0 \%$ & $24 \%$ \\
\hline Postfilialen & $35 \%$ & $42 \%$ & $2 \%$ & $0 \%$ & $21 \%$ \\
\hline Vereine & $13 \%$ & $11 \%$ & $7 \%$ & $48 \%$ & $22 \%$ \\
\hline Dorfgemeinschaftshäuser & $31 \%$ & $7 \%$ & $7 \%$ & $35 \%$ & $22 \%$ \\
\hline Einkaufsgelegenheiten & $16 \%$ & $\mathbf{6 3 \%}$ & $1 \%$ & $3 \%$ & $17 \%$ \\
\hline
\end{tabular}

Quelle: Neu et al. 2007; eigene Erhebung. 
Die Einschätzung der Daseinsvorsorgekompetenzen durch die Befragten der Gemeinde Galenbeck lässt sich bei typisierender Betrachtung in drei Kategorien fassen: Die erste Kategorie enthält die Daseinsvorsorgeleistungen, die nach einer absoluten Mehrzahl der befragten Personen bei staatlichen Stellen liegen sollen. Hierunter fallen die Gewährleistung der öffentlichen Sicherheit, des Schulangebots sowie technischer Infrastrukturen wie Straßenbau sowie Wasserversorgung und -entsorgung, die zwar mit 49 Prozent prozentual etwas abgeschlagen, aber dennoch der ersten Kategorie zurechenbar ist (in der dimap-Haushaltskundenbefragung wünschten sich gar 75 Prozent der Befragten, dass die Trinkwasserversorgung nicht in privaten Händen läge). Die zweite Kategorie umfasst die Daseinsvorsorgesektoren, die nach einer "nur " relativen Mehrheit der Befragten überwiegend von staatlichen Akteuren zu verantworten sein sollten. Zu dieser Gruppe gehören finanzielle, soziale und kulturelle Angebote wie Bankfilialen, Feuerwehr, Kindergärten, öffentlicher Personennahverkehr (ÖPNV), Schwimmbäder sowie die Seniorenbetreuung. Wesentlich bei der Bewertung dieser zweiten Kategorie ist jedoch, dass die absolute Mehrheit der Befragten diese Daseinsvorsorgeleistungen jedenfalls nicht (mehr) notwendigerweise dem Staat zuordnet. Dies zeigt sich gerade im Hinblick auf die Einschätzung des ÖPNV, bei dem 40 Prozent der Befragten eine staatliche, zugleich 38 Prozent der Befragten eine privatunternehmerische Leistungserfüllung für möglich halten. Analoges gilt für die Gewährleistung der Feuerwehr im Verhältnis von staatlicher Leistungserbringung und bürgerschaftlichem Engagement, wobei sich hier die traditionelle Unterscheidung zwischen verbeamteter Berufsfeuerwehr und freiwilliger Feuerwehr widerspiegelt. Darüber hinaus bleibt allerdings festzustellen, dass gerade privaten Unternehmen in erster Linie in denjenigen Daseinsvorsorgesektoren Kompetenzen zugesprochen werden, in denen sie schon immer tätig waren oder mittlerweile üblicherweise tätig sind. Die dritte Kategorie beinhaltet die Leistungsfelder der Daseinsvorsorge, in denen auch keine relative Mehrheit eine staatliche Trägerschaft für erforderlich hält. Dieser Kategorie werden die ärztliche Versorgung, der Postversand und der Unterhalt von Dorfgemeinschaftshäusern sowie traditionell gesellschaftliche Bereiche wie das Vereinsleben und die Schaffung von Einkaufsmöglichkeiten zugerechnet. Freie Träger spielen - mit Ausnahme der Seniorenbetreuung - aus Sicht der befragten Einwohner Galenbecks eine untergeordnete Rolle bei der Aufgabenzuordnung. ${ }^{48}$

Es lässt sich also aufgrund der empirischen Erhebungen feststellen, dass die Bürgerinnen und Bürger - im Gegensatz zu Forstoffs Diagnose - in der Daseinsvorsorge keine staatliche Monopolveranstaltung mehr sehen, sondern bereit sind, zwischen verschiedenen Daseinsvorsorgesektoren zu unterscheiden: Eine Grundversorgung mit Sicherheit, Schulen und technischen Infrastrukturen (Energie, Straße, Wasser) soll vom Staat erbracht werden. Darüber hinaus zeigen sich die Bürger aber für eine bürgerliche, freie und/oder unternehmerische Leistungserbringung auf den Daseinsvorsorgesektoren Bank, Gesundheit, Kultur und Post sowie Feuerwehr und Gemeinschaftseinrichtungen bereit. 


\section{Bedarfsstruktur der Daseinsvorsorge}

Die Bedarfsstruktur der Daseinsvorsorge reflektiert hingegen die Bedeutung, die die Bürgerinnen und Bürger den einzelnen Infrastrukturbereichen beimessen. Insoweit zeigen empirische Erhebungen aus unterschiedlichen ländlichen Räumen, dass die Bürgerinnen und Bürger keineswegs alle Infrastruktursegmente gleich, sondern entsprechend ihrer Bedarfsstruktur hierarchisch abgestuft bewerten.

\section{Abbildung 4: Wahrgenommener Bedarf an lokalen Dienstleistungen und Infra- struktur in der Gemeinde Galenbeck}

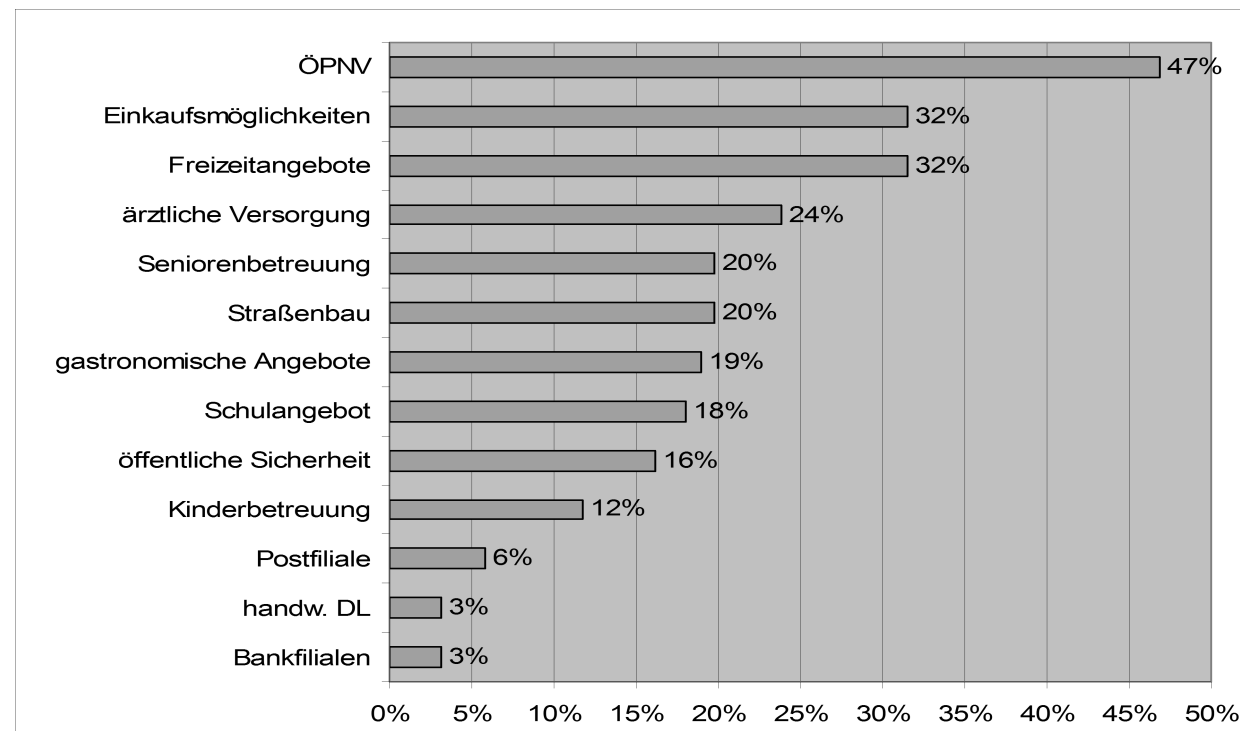

Quelle: Neu et al. 2007, S. 38; eigene Erhebung.

Frage: Welche der folgenden drei Bereiche sollten Ihrer Meinung nach in den kommenden Jahren verstärkt angeboten werden?

Nicht nur in den Erhebungen aus dem ländlichen Mecklenburg-Vorpommern (Abbildungen 4 und 5) stehen die ärztliche Versorgung, die Kinder- und Seniorenbetreuung und das Schulangebot, der ÖPNV sowie die Lebensmittelversorgung an der Spitze der als notwendig erachteten Daseinsvorsorgebereiche, sondern auch in Vergleichsuntersuchungen aus dem Landkreis Rotenburg (Wümme), einer ländlichen Region zwischen Bremen und Hamburg, oder der Hocheifel. In den beiden westdeutschen Regionen steht der ÖPNV an erster Stelle, dann folgen auf den weiteren Plätzen ärztliche Versorgung, Einkaufsmöglichkeiten und in der Hocheifel auch der Internetzugang. Die Ergebnisse einer Umfrage des Bundesinstituts für Bau-, 
Abbildung 5: Zukünftiger Infrastrukturbedarf im ländlichen Raum MecklenburgVorpommern (Mela-Befragung)

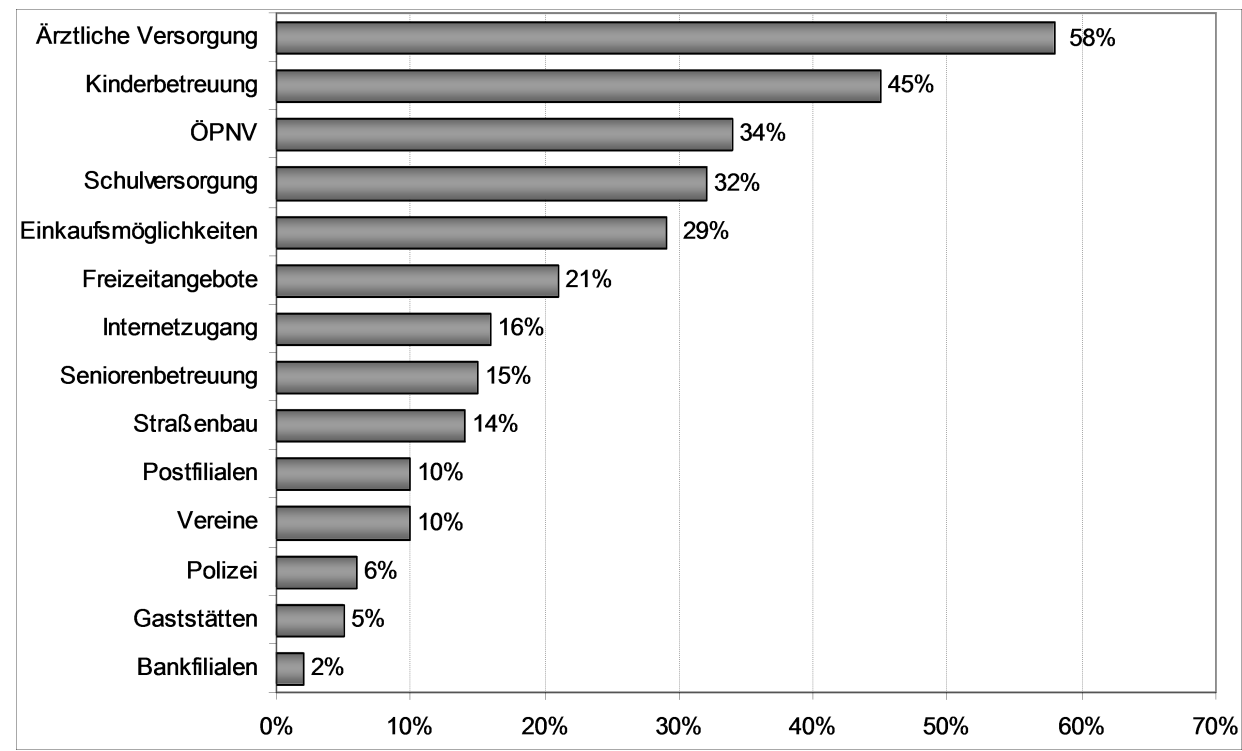

Quelle: Ickert et al. 2009, S. 9; eigene Erhebung.

Stadt- und Raumforschung zur Lebensqualität in kleinen Städten und Landgemeinden weisen in die gleiche Richtung. ${ }^{49}$

Mithin wird dem öffentlichen Personennahverkehr, der Gesundheitsversorgung wie sozialer und kultureller Infrastruktur besonderes Gewicht beigemessen, während den Gewährleistungen öffentlicher Sicherheit sowie technischen Infrastrukturangeboten nur nachgeordnete Bedeutung zukommt. Aus diesen Erhebungen lässt sich ablesen, dass sich die konkrete Bedarfsstruktur vor allem an den Lebensverhältnissen vor Ort orientiert und nicht als die erwünschte Form der infrastrukturellen Grundversorgung verstanden werden darf, deren Prioritäten - Sicherheits-, Bildungs- und Technikinfrastruktur - in der staatlichen Verantwortungszuordnung zum Ausdruck kommen. Vielmehr liegt die Annahme nahe, dass diese Grundversorgung (noch) als staatlicherseits zu erbringende Selbstverständlichkeit aufgefasst wird, die den infrastrukturellen Hintergrund der jeweils konkreten Bedarfsstruktur bildet. Darüber hinaus fühlen sich viele Befragte auch nicht durch die Appelle an den "aktiven Bürger " angesprochen. Offensichtlich dominiert die Auffassung, dass durch Abgaben und Steuern bereits ein angemessener Beitrag zur Sicherung von Infrastrukturen geleistet wird. Dies allerdings bedeutet, dass die Chancen auf mehr bürgerliche Mitwirkung bei der daseinsvorsorgenden Leistungserbringung unter den gegebenen Umständen nicht gut stehen. Der Rückbau der infrastrukturellen

49 Vgl. Neu, Nikolic 2013; Ickert et al. 2009; BBSR 2011, S. 14.

Leviathan, 40. Jg., 4/2012 
Grundversorgung schwächt die Rahmenbedingungen von Zivilgesellschaft. Bürgerinnen und Bürger kommen sich bei der Gewährleistung der Daseinsvorsorge eher als »Ausfallbürgen « vor und weniger als Partner. ${ }^{50}$

\section{4. »Neue Sichtbarkeit« der Infrastrukturen}

Wie groß die sozialen und territorialen Integrationsleistungen in den vergangenen Jahrzehnten waren, zeigt sich erst, wenn die öffentlichen Infrastrukturen erodieren oder wegfallen. Doch eine Neukonzeption der Daseinsvorsorge kann unter den derzeitigen demografischen Bedingungen, der Schuldenlast von Bund und Gemeinden sowie der globalen Finanzkrise nicht mehr ausschließlich auf den Staat als Leistungserbringer setzen. Sie darf aber auch nicht allein auf das private Engagement vertrauen, da die Bürgerinnen und Bürger durch eine vollkommen eigenständige Gewährleistung »ihrer " Daseinsvorsorge schnell überfordert wären. Deshalb muss eine Neukonzeption erstens zu einer differenzierten Verantwortungsteilung für die Daseinsvorsorge zwischen staatlichen, kommunalen und privatwirtschaftlichen sowie zivilgesellschaftlichen Akteuren finden und zweitens die langfristigen Infrastrukturgefälle in Rechnung stellen, welche die Bundesrepublik als eine sozialräumlich zugleich schrumpfende und wachsende Gesellschaft prägen. Mit einer solchen Neukonzeption wäre ebenso eine neue Sichtbarkeit der Infrastrukturen verbunden, die der bundesrepublikanischen Gesellschaft den sozialen, politischen und wirtschaftlichen Wert ihrer infrastrukturierten Daseinsvorsorge vor Augen führt.

\subsection{Leistungsbezogene Differenzierung der Daseinsvorsorge}

Besonders die Kommunen in peripheren ländlichen Räumen stehen vor der Herausforderung, angesichts von Schrumpfung, Alterung, geringer Steuereinnahmen und hohem kommunalen Schuldenstand das gewohnte steigende Infrastrukturniveau auch weiterhin anzubieten. Diese Kommunen sehen ihre finanziellen Handlungsspielräume schwinden. Das Bemühen, neue Partner bei der daseinsvorsorgenden Leistungserstellung zu gewinnen, stößt an enge Grenzen, denn gleichzeitig sind die Bürgerinnen und Bürger nicht in der Lage, insbesondere die technischen Infrastrukturen in vollkommen eigener Regie zu betreiben. Deshalb muss eine infrastrukturelle Neukonzeption auf eine innovative Verantwortungsteilung zwischen staatlichen, privatwirtschaftlichen und zivilgesellschaftlichen Akteuren setzen, ${ }^{51}$ die den Wandel des Verhältnisses der Verantwortungs- und der Bedarfsstruktur der Daseinsvorsorge reflektiert. Die empirischen Erhebungen haben gezeigt, dass die Bürgerinnen und Bürger die infrastrukturierte Daseinsvorsorge nicht (mehr) als einen monolithischen Block verstehen. Vielmehr differenzieren sie zwischen den einzelnen Daseinsvorsorgesektoren, welche im Hinblick auf die Verantwortungsperspektive der Leistungserstellung nach den Prioritäten sozialer, politischer und

50 Vgl. Neu et al. 2007, S. 70 ff.

51 Vgl. grundsätzlich zum Konzept der Verantwortungsteilung in der Daseinsvorsorge Schuppert 2002, S. 14 ff. 
wirtschaftlicher Integration und in der Bedarfsperspektive nach den alltäglichen Nöten der Bürgerinnen und Bürger hierarchisiert werden.

Dabei lassen sich die einzelnen Infrastruktursektoren drei verschiedenen Typen der staatlichen Aufgabenerfüllung zuordnen: dem »Leistungsstaat «, dem »Gewährleistungsstaat « und dem » motivierenden Staat «. Diese drei Staatsbilder wurden und werden - zusammen mit weiteren Staatstypen wie dem dienstleistenden, dem schlanken, dem aktivierenden oder dem ermöglichenden Staat ${ }^{52}$ - oft als Substitute verstanden, etwa in dem Sinn, dass der Leistungsstaat durch den Gewährleistungsstaat "abgelöst « worden sei. Doch diese theoretische Vorstellung geht an der politischen Wirklichkeit vorbei: Der Staat setzt leistungsstaatliche, gewährleistungsstaatliche und motivierende Instrumente parallel ein, um das Gemeinwohl zu garantieren. Dies gilt auch für die Daseinsvorsorge: Als Leistungsstaat verantwortet die öffentliche Hand die Sicherheits-, Schul- und Verkehrsinfrastrukturen unmittelbar selbst: im Fall der Sicherheit als flächendeckendes staatliches Gewaltmonopol, im Fall der Schulen wegen des grundgesetzlichen demokratischen Bildungsauftrags für die soziale, politische und wirtschaftliche Zukunft der Bundesrepublik und im Fall der Verkehrsinfrastruktur aufgrund deren Bedeutung für eine gerade in flächigen Schrumpfungsregionen automobilzentrierte Mobilität. Als Gewährleistungsstaat garantiert das Gemeinwesen vor allem die technischen Infrastrukturen: Das Regulierungsrecht gewährleistet die Universaldienste in den Bereichen der Telekommunikation, der Post sowie der Energieversorgung. Die vergleichsweise höheren Kosten für die Versorgung von Schrumpfungsregionen werden von den Telekommunikations-, Post- und Energieversorgungsunternehmen übernommen, weil der überaus lukrative Gesamtauftrag der Versorgung der Republik sonst gefährdet wäre. ${ }^{53} \mathrm{Als}$ motivierender Staat fördert die öffentliche Hand den ÖPNV, ${ }^{54}$ die (umstrittene) Neumodellierung der medizinischen Versorgungsstruktur ${ }^{55}$ sowie Selbstorganisationsformen für die Kinder- und Seniorenbetreuung in schrumpfenden Regionen. ${ }^{56}$

Mit diesen unterschiedlichen Rollen des Staats korrespondieren differenzierte Einbindungen von Wirtschaftsunternehmen (Regulierung) sowie der Bürgerinnen und Bürger (Motivierung) in die Erbringung von technischen und sozialen Infrastrukturleistungen. Damit ist zugleich der ganz wesentliche Effekt verbunden, dass die soziale, politische und wirtschaftliche Bedeutung der Infrastrukturleistungen für die Gesellschaft sichtbar wird: Die geräuschlose »Vorsorgemaschine « 57 des Wohlfahrtsstaats hatte die infrastrukturellen Daseinsvorsorgeleistungen durch »unsicht-

52 Vgl. zum Nutzen des Denkens in Staatstypen Schuppert 2000, S. 55 ff.

53 Vgl. Kersten 2006 b, S. 945 ff.

54 Vgl. das »Altmark Trans«-Urteil des EuGH vom 24. Juli 2003, NJW 2003, S. 2515 ff.

55 Vgl. Deutscher Bundestag 2011; zur streitigen Bundestagsdebatte siehe http://www.bundestag.de/dokumente/textarchiv/2011/36854449_kw48_de_gkv/index.html (Zugriff vom 30.12.2011).

56 Vgl. zur Bereitstellungsfunktion des Rechts Burgi 2006, $\mathbb{1 8}$, Rn. 35.

57 Kaube 2002, S. 34.

Leviathan, 40. Jg., 4/2012 
bare öffentliche Hände « 58 erbracht. Eine "geräuschlose « Neuverteilung der Infrastrukturverantwortung ist in einer pluralistischen Gesellschaft jedoch schon aufgrund der damit verbundenen harten Konflikte um die ökonomischen Folgekosten nicht zu erwarten. In den sozialen Auseinandersetzungen um die Neuverteilung der gesellschaftlichen Infrastrukturverantwortung müssen alle individuellen wie kollektiven, öffentlichen wie privaten, gemeinwohlverpflichteten wie ökonomischen Akteure einschätzen und aushandeln, wie viel ihnen die infrastrukturelle Vernetzung »ihrer " Gesellschaft sozial, politisch und wirtschaftlich wert ist. Die einzelnen Infrastrukturbereiche stehen jeder für sich auf dem Prüfstand - Strom, Wasser, Müllabfuhr, Winterdienst, Freibäder und der ÖPNV. Wer soll und kann zuständig sein? Zu welchen Preisen? Brauchen wir eine stärkere Rekommunalisierung? Oder geht es auch mit dem Bürgerbus? Dies führt zu einer neuen Sichtbarkeit der Infrastrukturen, die nicht mehr als selbstverständlich vorhanden vorausgesetzt, sondern als gestaltbar erlebt werden. Auf diesem Weg kann die bundesrepublikanische Gesellschaft zu einem neuen Gleichgewicht zwischen Grundversorgung und infrastrukturellen Ligaturen, verstanden als Daseinsvorsorgeleistungen, die je nach Bedarf vor Ort »zugespielt « werden, finden. In diesem konfliktreichen Prozess wird einerseits öffentlichen und privaten Wirtschaftsunternehmen Infrastrukturverantwortung aktiv zugeordnet werden (Universaldienste), andererseits können Bürgerinnen und Bürger aktiv motiviert werden, individuell wie kollektiv als Privatpersonen Infrastrukturverantwortung zu übernehmen. Wo und wie auf diese Weise ein neuer Infrastrukturvertrag zwischen Bürgerinnen und Bürgern, Wirtschaftsunternehmen und öffentlicher Hand gelingt, wird maßgeblich davon abhängen, wie ausgeprägt der politische Mut ist, Infrastrukturkonflikte offen auszutragen, Lösungsvorschläge zwischen allen Beteiligten neu zu verhandeln sowie Handlungsspielräume und Eigenverantwortlichkeiten für neue Akteure zuzulassen. Infolge dieser neuen Sichtbarkeit der Infrastrukturen wird ein soziales, politisches und wirtschaftliches Zeichen in Schrumpfungsregionen gesetzt. Ein solches Zeichen ist auch für die ganz bewusste Entfaltung der Zivilgesellschaft durch den Staat selbst wichtig: Soweit die Verwaltung Auswahlentscheidungen für die Besetzung von Personalstellen in der Verwaltung trifft, sollte sie auch mehr die sozialen Fähigkeiten einer Bewerberin oder eines Bewerbers berücksichtigen und sein bürgerschaftliches Engagement honorieren.

\subsection{Sozialräumliche Differenzierung der Daseinsvorsorge}

Diese Neuverhandlung des infrastrukturellen Gesellschaftsvertrags kann nicht isoliert in lokaler oder regionaler Perspektive erfolgen. Sie muss dabei nicht nur politisch sensibel die historisch gewachsene Verbindung von Infrastruktur, Daseinsvorsorge und Gleichwertigkeitsgrundsatz reflektieren, sondern insbesondere auch das Infrastrukturgefälle einbeziehen, das sich in der Bundesrepublik aufgrund des unmittelbaren Nebeneinanders von Schrumpfungs- und Wachstumsregionen ausdifferenziert hat. Die infrastrukturelle Grundfrage, wie lokale und regionale Infra- 
strukturen miteinander vernetzt sind, muss dahingehend erweitert werden, wie sich diese Vernetzung im nationalen und europäischen Kontext entfaltet. Die Beantwortung dieser Frage zielt auf das Leitbild, dem die Vernetzung der infrastrukturierten Daseinsvorsorge folgt. Die Formulierung eines solchen Leitbilds wird im Hinblick auf die infrastrukturelle Verantwortungsteilung umso notwendiger, als nun nicht mehr »nur « der Wohlfahrtsstaat, sondern eine Vielzahl von staatlichen, wirtschaftlichen und zivilgesellschaftlichen Akteuren auf lokaler, regionaler, nationaler und europäischer Ebene für die Gestaltung und Erbringung von infrastrukturierten Daseinsvorsorgeleistungen verantwortlich sein sollen. Diese gemischte Infrastrukturverantwortung wird nicht mehr zentral gesteuert und ist deshalb auf ein gemeinsames Leitbild angewiesen, um die lokale, regionale, nationale und europäische Infrastrukturentwicklung zu koordinieren.

Das infrastrukturelle Leitbild der Daseinsvorsorge des Wohlfahrtsstaats bildet die Gleichwertigkeit bzw. die Einheitlichkeit der Lebensverhältnisse, die sich normativ an Artikel 72 Absatz 2 und Artikel 106 Absatz 3 Satz 4 Nummer 2 des Grundgesetzes anlehnen. Doch unter den Bedingungen des ökonomischen und demografischen Wandels und der damit einhergehenden Infrastrukturkrise verblasst dieses Leitbild der Gewährleistung der flächendeckenden Daseinsvorsorge auf einem hohen wohlfahrtsstaatlichen Leistungsniveau und verliert auch seine normative Steuerungskraft. ${ }^{59}$ Dies spiegelt sich in der Rechtsprechung des Bundesverfassungsgerichts zu dem 1994 neu gefassten Artikel 72 Absatz 2 GG wider: Die Formel von der » Gleichwertigkeit der Lebensverhältnisse im Bundesgebiet « im Sinne des Artikel 72 Absatz 2 GG soll nach Auffassung der Karlsruher Richter »nur " noch dann einschlägig sein, »wenn sich die Lebensverhältnisse in den Ländern der Bundesrepublik in erheblicher, das bundesstaatliche Sozialgefüge beeinträchtigender Weise auseinander entwickelt haben oder sich eine derartige Entwicklung konkret abzeichnet «. ${ }^{60}$ Verfassungsrechtlich wird mit dem Begriff der » Gleichwertigkeit der Lebensverhältnisse " also nur noch das "Minimum " als die unterste Schwelle des sozialen Zusammenhalts in der Bundesrepublik beschrieben. Mit dieser Neubestimmung der Gleichwertigkeit der Lebensverhältnisse deutet das Bundesverfassungsgericht zwar die Richtung an, in der sich nach einer neuen Leitvorstellung suchen lässt, um die demografische Herausforderung für die Gestaltung der Daseinsvorsorge anzunehmen: Es geht darum, auf der Grundlage eines Mindeststandards des sozialen Zusammenhalts eine Leitvorstellung für eine räumlich differenzierte Ausgestaltung der Infrastruktur zu entwickeln. Doch die Grenzen der normativen Steuerungskraft der neuen Karlsruher Begriffsbestimmung für die Gestaltung der Daseinsvorsorge in einer schrumpfenden Gesellschaft sind eben auch deutlich: Liegt das Problem der tradierten Bestimmung des Gleichwertigkeitspostulats in der einseitigen Wachstumsorientierung auf ein hohes wohlfahrtsstaatliches Niveau, so liegt das Problem der Karlsruher Neuinterpretation des Gleichwertigkeitsgrundsatzes in der einseiti-

59 Vgl. hierzu und zum Folgenden Kersten 2006 a, S. 249 ff.; Kersten 2006 b, S. 945 ff.; a.A. Blotevogel 2006, S. 464.

60 Vgl. BVerfGE 106, 62 (144 ff.); 110, 141 (174 ff.); 111, 10 (28f.); 111, 226 (253 ff.); 112,226 (244). 
gen (Rückbau-)Orientierung auf ein Minimum der Daseinsvorsorge. Deshalb ist das Gleichwertigkeitspostulat so oder so nicht in der Lage, normativ auf das Infrastrukturgefälle zwischen Wachstums- und Schrumpfungsregionen zu reflektieren, welche die Gewährleistung der Daseinsvorsorge gegenwärtig und künftig bestimmen.

Für die normative Konturierung eines neuen Leitbilds für die Gestaltung der infrastrukturierten Daseinsvorsorge lohnt sich ein Blick auf die europäische Ebene, die mit sehr großen sozialräumlichen Gefällen umgehen muss. Dafür folgt die Verfassungsstruktur der europäischen Verträge keineswegs - wie von Jürgen Habermas favorisiert ${ }^{61}$ - dem Grundsatz einheitlicher Lebensverhältnisse, sondern hat nurmehr das Leitbild des " wirtschaftlichen, sozialen und territorialen Zusammenhalts " als Unionsziel entworfen. ${ }^{62}$ Jenseits des allgemeinen Kohärenzgebots für eine nachhaltige wirtschaftliche, soziale und territoriale Kohäsionspolitik (Artikel 7 AEUV Vertrag über die Arbeitsweise der Europäischen Union) integrieren die Europäischen Verträge das Unionsziel des wirtschaftlichen, sozialen und territorialen Zusammenhalts ausdrücklich in zwei Politikbereichen, aus denen sich die nähere Kontur des Leitbilds für die Ausgestaltung der infrastrukturierten Daseinsvorsorge ergibt: Die Struktur- und Regional- sowie die Transeuropäische Netzpolitik sind dem Grundsatz der wirtschaftlichen, sozialen und territorialen Kohäsion verpflichtet. ${ }^{63}$ Insbesondere in diesen Politikfeldern zeigt sich das normative Konzept des sozialen, wirtschaftlichen und territorialen Zusammenhalts, durch die Neuvernetzung von Infrastrukturen sozialräumliche Divergenzen zu überwinden. Es kommt beim Kohäsionskonzept darauf an, mittels dieser neuen Vernetzungen einen neuen, europäischen sozialen, wirtschaftlichen und territorialen Raum zu konstituieren und sichtbar zu machen. Hierfür bildet die soziale, wirtschaftliche und territoriale Kohäsion eine zentrale Zielsetzung der Dienste von allgemeinem wirtschaftlichen Interesse (Artikel 14 AEUV), wobei über die grundrechtlich fundierte Zugangsgarantie zu diesen raumgreifenden Daseinsvorsorgeeinrichtungen die freiheitliche Dimension des Kohäsionskonzepts unterstrichen wird. ${ }^{64}$ Eine diesem europäischen Leitbild des wirtschaftlichen, sozialen und territorialen Zusammenhalts folgende Infrastrukturentwicklung empfiehlt sich auch für die Fortschreibung des Gesellschaftsvertrags der Bundesrepublik, um die Daseinsvorsorge in einer sozialräumlich zugleich schrumpfenden und wachsenden Gesellschaft differenzierend zu gestalten: ${ }^{65}$ Das Leitbild toleriert in unterschiedlichen Teilräumen eine differenzierte Entwicklung der Daseinsvorsorge. Es fordert für die differenzierte Entwicklung der Daseinsvorsorge in diesen Teilräumen aber, dass sie sich funktional ergänzt, das heißt sich in die wirtschaftliche, soziale und territoriale Entwicklung des Gesamt-

61 Vgl. Kaube 2011.

62 Artikel 3 Absatz 3 Unterabsatz 3 Vertrag über die Europäische Union (EUV). Vgl. hierzu und zum Folgenden Kersten 2009, S. 27 ff.; Reichel 2009, S. 187 ff.

63 Artikel 174 Unterabsatz 1 in Verbindung mit Artikel 170 Absatz 1 AEUV.

64 Artikel 6 Absatz 1 EUV in Verbindung mit Artikel 36 Charta der Grundrechte der Europäischen Union.

65 Vgl. hierzu und zum Folgenden Kersten 2006 a, S. 249 ff.; Kersten 2006 b, S. 945 ff. 
raums integriert. Diese Integration orientiert sich an der Einsicht, dass die Bürgerinnen und Bürger in der freien Entfaltung ihrer Persönlichkeit in der liberalen Demokratie auf die Teilhabe an einer vernetzten und damit raumgreifenden Daseinsvorsorge angewiesen sind. Deshalb setzt das Leitbild auf den verschiedenen Daseinsvorsorgesektoren eine Grundversorgung voraus, die - ganz im Sinne der neuen Rechtsprechung des Bundesverfassungsgerichts zu Artikel 72 Absatz 2 GG die untere Grenze des sozialpolitischen Zusammenhalts der Bundesrepublik beschreibt. Bei der Definition dieser Grundversorgung kann aufgrund der räumlichen Differenzierung der Daseinsvorsorge in Gebieten mit einer schrumpfenden Gesellschaft nicht schlicht auf Durchschnittswerte gesetzt werden. Vielmehr sind die Differenzen zu reflektieren, die zwischen Zentren und Peripherien bestehen und die es anhand des verfassungsrechtlichen Maßstabs der freien Entfaltung der Persönlichkeit aller Bürgerinnen und Bürger in der liberalen Demokratie "aufzuheben " gilt. Das Leitbild der Gleichwertigkeit der Lebensverhältnisse wird also keineswegs aufgegeben, sondern bildet die Grundlage, auf der sich das Konzept des sozialen, wirtschaftlichen und territorialen Zusammenhalts ausdifferenzieren kann.

\section{Fazit}

Die Effekte des demografischen Wandels sind keineswegs allein auf die sozialen Sicherungssysteme begrenzt. Vielmehr kommen mit der Demografiedebatte die Voraussetzungen des sozialen und territorialen Zusammenhalts der bundesrepublikanischen Gesellschaft auf die wissenschaftliche, zeitdiagnostische und politische Tagesordnung. Denn über lange Jahrzehnte hinweg bestimmte das politische Leitbild, die Lebensverhältnisse zwischen Stadt und Land, Nord und Süd, Ost und West anzugleichen, den Auf- und Ausbau der öffentlichen Infrastrukturen. Geburtenrückgang, Wanderungsbewegungen und rasante Alterung, flankiert von Finanzkrise und hoher Staatsverschuldung, entziehen diesem Leitbild freilich aktuell mehr und mehr die Grundlage. Die De-Infrastrukturalisierung ganzer (peripherer) Regionen ist nicht mehr zu dementieren. Die Folgen dieses Um- und Abbaus sozialer und technischer Infrastrukturen sind in vielen Gemeinden spürbare Versorgungsengpässe, weitreichende Verluste an politischer Öffentlichkeit und die anhaltende Auszehrung demokratischer Staatlichkeit. Die Antwort auf diese demografische Provokation liegt nach unserer Ansicht in einer neuen Verantwortungsteilung von Staat, Wirtschaft und Zivilgesellschaft für die infrastrukturierte Daseinsvorsorge. Wird auf diesem Wege nach innovativen, bedarfsgerechten Lösungen gesucht, die auch weiterhin dem infrastrukturellen Leitmotiv »Integration über Infrastrukturen « verpflichtet sind, so werden sich auch die staatlichen Institutionen verändern müssen. Eine Flexibilisierung der Angebotsformen, die nicht allein am Minimum, sondern am lokalen Bedarf orientiert sind, wird ebenso notwendig sein wie eine Priorisierung der öffentlichen Leistungen. Einen gangbaren Weg zur Umsetzung dieser neuen Infrastrukturgestaltung bietet die Differenzierung zwischen den unterschiedlichen Daseinsvorsorgebereichen und Infrastruktursektoren: Dabei kommt vor allem staatlichen und kommunalen Einrichtungen die Aufgabe zu, selbst oder durch die 
rechtliche Verpflichtung privater Unternehmen eine daseinssichernde Grundversorgung flächendeckend zu gewährleisten. Welche infrastrukturellen Leistungen neben dieser Grundversorgung (vor Ort) noch anzubieten sind und von wem, um Zugang und Teilhabe am gesellschaftlichen Leben zukünftig zu sichern, wird von Staat, Wirtschaft und Zivilgesellschaft in jedem Einzelfall ausgehandelt und gestaltet werden müssen. Hier sind zweifelsohne Infrastrukturkonflikte programmiert, zu deren Lösung es politischer Kreativität bedarf. Das gilt für alle Bereiche: für die Gestaltung der individuellen und öffentlichen Mobilität, für den Umbau der lokalen Energieversorgung, aber auch für die Neustrukturierung des Bildungs- und Gesundheitswesens. Auf der Grundlage infrastruktureller Konflikte und Neuverteilungen könnte sich ein Vereins- und Gemeindeleben entfalten, das dennoch nach wie vor staatliche Unterstützung und professionelles Verwaltungspersonal benötigt. Neuverteilung und Neujustierung von Infrastrukturleistungen bedeutet nicht Entstaatlichung. Aber es bedarf anderer und größerer politischer, rechtlicher und finanzieller Handlungsspielräume, um neue Mitspieler zu gewinnen. Gelänge es, die demografische Herausforderung anzunehmen und nicht, wie bisher geschehen, weitgehend zu ignorieren, dann stünde auch der soziale, wirtschaftliche und territoriale Zusammenhalt der Bundesrepublik nicht zur Disposition. Denn nur das Zusammenspiel von engagierter Bürgergesellschaft und leistungsbereiter Staatlichkeit ist der Garant dafür, dass sich aus der demografisch induzierten Infrastrukturkrise keine Krise der rechts- und sozialstaatlich geprägten Demokratie entwickelt.

\section{Literatur}

Albrecht, Peter-Georg 2009. ») Wenn jemand fragt, wir würden das machen ...<- Engagementpotenziale junger Senioren in ländlichen Räumen Ostdeutschlands ", in Daseinsvorsorge. Eine gesellschaftswissenschaftliche Annäherung, hrsg. v. Neu, Claudia, S. 206-219. Wiesbaden: VS Verlag für Sozialwissenschaften.

Barlösius, Eva et al. 2011. »Infrastrukturen neu denken: gesellschaftliche Funktionen und Weiterentwicklung ", in Globaler Wandel und regionale Entwicklung. Anpassungsstrategien in der Region Berlin-Brandenburg, hrsg. v. Hüttl, Reinhard et al., S. 147-173. Berlin: Springer.

Battis, Ulrich; Kersten, Jens; Mitschang, Stephan 2010. »Klimaschützende Stadterneuerung «, in Zeitschrift für Gesetzgebung 25, 3, S. 247-259.

BBR (Bundesamt für Bauwesen und Raumordnung) 2007. Regionalplanerische Handlungsansätze zur Gewährleistung der öffentlichen Daseinsvorsorge, MORO-Informationen 2/2. Bonn.

BBSR (Bundesinstitut für Bau-, Stadt- und Raumforschung) 2011. Lebensqualität in kleinen Städten und Landgemeinden. Bonn.

Beauftragter der Bundesregierung für die Neuen Länder 2011. Daseinsvorsorge im demografischen Wandel zukunftsfähig gestalten. Handlungskonzept zur Sicherung der privaten und öffentlichen Infrastruktur in vom demografischen Wandel besonders betroffenen ländlichen Räumen. Berlin.

Becker, Udo et al. 2001. Postfossile Mobilität und Raumentwicklung. Positionspapier Nr. 89 aus der Akademie für Raumforschung und Landesplanung (ARL). Hannover: ARL.

BiB (Bundesinstitut für Bevölkerungsforschung) 2011. Lebenserwartung von Ruheständlern so hoch wie nie zuvor. http://www.bib-demografie.de/nn_749852/DE/Home/Grafik_des_M onats/Archiv/2011/2011_03_maerz.html (Zugriff vom 09.09.2012).

Blotevogel, Hans H. 2006. »Neuorientierung der Raumordnungspolitik? Die neuen >Leitbilder und Handlungsstrategien für die Raumentwicklung in Deutschland in der Diskussion ", in Raumforschung und Raumordnung 64, 6, S. 460-472. 
BMELV (Bundesministerium für Ernährung, Landwirtschaft und Verbraucherschutz) 2008. So haben ländliche Räume Zukunft. Ergebnisse und Erfahrungen des Modellvorhabens REGIONEN AKTIV. Berlin.

BMELV 2012. Modellprojekt LandZukunft. http://www.land-zukunft.de (Zugriff vom 03.08.2012).

BMI (Bundesministerium des Innern) 2012. Jedes Alter zählt. Demografiestrategie der Bundesregierung. Berlin.

BMVBW (Bundesministerium für Verkehr, Bau- und Wohnungswesen); BBR (Bundesamt für Bauwesen und Raumordnung). Hrsg. 2005. Öffentliche Daseinsvorsorge und demographischer Wandel. Berlin, Bonn.

Burgi, Martin 2006. "Rechtsregime", in Grundlagen des Verwaltungsrechts, Band 1, hrsg. v. Hoffmann-Riem, Wolfgang; Schmidt-Aßmann, Eberhard; Voßkuhle, Andreas, S. 1175-1236. München: C. H. Beck.

Canzler, Weert; Knie, Andreas 2009. »Auf dem Weg zum Gewährleistungsstaat. Netzvermarktung und Infrastrukturpolitik für die schrumpfende Gesellschaft «, in Daseinsvorsorge. Eine gesellschaftswissenschaftliche Annäherung, hrsg. v. Neu, Claudia, S. 97-111. Wiesbaden: VS Verlag für Sozialwissenschaften.

Castel, Robert 2005. Die Stärkung des Sozialen. Hamburg: Hamburger Edition.

Dahrendorf, Ralf 2004 [1995]. »Die Quadratur des Kreises: Wirtschaftlicher Wohlstand, sozialer Zusammenhalt und politische Freiheit", in Ralf Dabrendorf: Der Wiederbeginn der Geschichte, S. 103-131. München: C. H. Beck.

Davy, Benjamin 2007 a. »Bodenpolitik in der leeren Stadt", in Flächenmanagement und Bodenordnung 69, 2, S. 56-64.

Davy, Benjamin 2007 b. »Was kostet die Leere? «, in Revitalisierender Städtebau, hrsg. v. Sulzer, Jürg, S. 138-147. Dresden: TUDpress.

Deutscher Bundestag 2011. Entwurf eines Gesetzes zur Verbesserung der Versorgungsstrukturen in der gesetzlichen Krankenversicherung (GKV-Versorgungsstrukturgesetz - GKV-VStG). Drucksache 17/6906. http://dipbt.bundestag.de/dip21/btd/17/069/1706906.pdf (Zugriff vom 10.10.2012).

dimap 2008. Haushaltskundenbefragung im Auftrag des Verbands der kommunalen Unternehmen. Bonn.

Forsthoff, Ernst 1938. Die Verwaltung als Leistungsträger. Stuttgart: Kohlhammer.

Forsthoff, Ernst 1941. Grenzen des Rechts. Königsberg: Gräfe \& Unzer.

Frey, René L. 2005. »Infrastruktur ", in Handwörterbuch der Raumordnung, 4. Auflage, hrsg. v. der Akademie für Raumforschung und Landesplanung, S. 469-457. Hannover: Verlag der ARL.

Gegner, Martin 2007. "Verkehr und Daseinsvorsorge «, in Handbuch der Verkehrspolitik, hrsg. v. Schöller, Oliver; Canzler, Weert; Knie, Andreas, S. 455-470. Wiesbaden. VS Verlag für Sozialwissenschaften.

Graham, Stephen; Marvin, Simon 2001. Splintering urbanism: networked infrastructures, technological mobilities and the urban condition. London, New York: Routledge.

Habermas, Jürgen 1990 [1962]. Strukturwandel der Öffentlichkeit. Untersuchungen zu einer Kategorie der bürgerlichen Gesellschaft. Neuwied: Suhrkamp.

Hermes, Georg 1998. Staatliche Infrastrukturverantwortung. Tübingen: Mohr Siebeck.

Ickert, Hannes; Neu, Claudia; Schröder, Marlen 2009. Infrastrukturbedarfe für den ländlichen Raum Mecklenburg-Vorpommerns - Ergebnisse der Besucherbefragung auf der Mecklenburgischen Landwirtschaftsausstellung 2008, hrsg. v. Ministerium für Landwirtschaft, Umwelt und Verbraucherschutz Mecklenburg-Vorpommern. Schwerin.

Jellinghaus, Lorenz 2006. Zwischen Daseinsvorsorge und Infrastruktur. Zum Funktionswandel von Verwaltungswissenschaften und Verwaltungsrecht in der zweiten Hälfte des 19. Jahrbunderts. Frankfurt: Klostermann.

Jhering, Rudolf von 1894. Geist des römischen Rechtes auf den verschiedenen Stufen seiner Entwicklung, 2. Theil, 1. Abteilung. 5. Auflage. Leipzig: Breitkopf \& Härtel.

Jünger, Ernst 1978 [1939]. »Auf den Marmorklippen «, in Ernst Jünger: Sämtliche Werke, Band 15, S. 247-351. Stuttgart: Klett-Cotta.

Kaube, Jürgen 2002. »Die Vorsorgemaschine«, in Frankfurter Allgemeine Zeitung, 14. September 2002.

Leviathan, 40. Jg., 4/2012 
Kaube, Jürgen 2011. »Hinter verschlossenen Türen «, in FAZ online. http://www.faz.net/artikel/ C31399/europa-in-der-krise-hinter-verschlossenen-tueren-30442112.html (Zugriff vom 08. 09.2011).

Kersten, Jens 2005. »Die Entwicklung des Konzepts der Daseinsvorsorge im Werk von Ernst Forsthoff ", in Der Staat 44, 4, S. 543-569.

Kersten, Jens 2006 a. »Abschied von der Gleichwertigkeit der Lebensverhältnisse - der 'wirtschaftliche, soziale und territoriale Zusammenhalt< als neue Leitvorstellung für die Raumplanung ", in Umwelt- und Planungsrecht 26, 7, S. 245-252.

Kersten, Jens 2006 b. »Universaldienste in einer schrumpfenden Gesellschaft «, in Deutsches Verwaltungsblatt 121,15, S. 942-949.

Kersten, Jens 2008. "Mindestgewährleistungen im Infrastrukturrecht «, in Informationen zur Raumentwicklung 1/2, S. 1-15.

Kersten, Jens 2009. »Wandel der Daseinsvorsorge ", in Daseinsvorsorge. Eine gesellschaftswissenschaftliche Annäherung, hrsg. v. Neu, Claudia, S. 22-38. Wiesbaden: VS Verlag für Sozialwissenschaften.

Kersten, Jens; Neu, Claudia; Vogel, Berthold 2012 a. »Demographische De-Infrastrukturalisierung ", in Zeitschrift für Agrargeschichte und Agrarsoziologie 60, 1, S. 42-59.

Kersten, Jens; Neu, Claudia; Vogel, Berthold 2012 b. Demografie und Demokratie. Hamburg: Hamburger Edition.

Laak, Dirk van 1999. » Der Begriff der >Infrastruktur und was er vor seiner Erfindung besagte «, in Archiv für Begriffsgeschichte XLI, S. 280-299.

Laak, Dirk van 2010. Das »vergrabene Kapital « und seine Wiederentdeckung. Das neue Interesse an der Infrastruktur. Berlin: Berlin-Brandenburgische Akademie der Wissenschaften.

Lange, Bastian; Matthies, Ulf 2005. »Raumpioniere - Konzeption, Prozesse, Netzstrukturen «, in Schrumpfende Städte. Band 2: Handlungskonzepte, hrsg. v. Oswalt, Philipp, S. 374-383. Ostfildern: Hatje Cantz.

Marx, Karl; Engels, Friedrich 1971 [1848]. "Kommunistisches Manifest ", in: Karl Marx: Die Frühschriften, hrsg. v. Landshut, Siegfried, S. 525-560. Stuttgart: Körner.

Meinel, Florian 2011. Der Jurist in der industriellen Gesellschaft. Ernst Forsthoff und seine Zeit. Berlin: Akademie Verlag.

Neu, Claudia 2006. »Territoriale Ungleichheit - eine Erkundung ", in Aus Politik und Zeitgeschichte 37, S. 8-15.

Neu, Claudia 2009. »Daseinsvorsorge und territoriale Ungleichheit ", in Daseinsvorsorge. Eine gesellschaftswissenschaftliche Annäherung, hrsg. v. Neu, Claudia, S. 80-96. Wiesbaden: VS Verlag für Sozialwissenschaften.

Neu, Claudia et al. 2007. Daseinsvorsorge im peripheren ländlichen Raum - am Beispiel der Gemeinde Galenbeck, hrsg. v. Ministerium für Landwirtschaft, Umwelt und Verbraucherschutz Mecklenburg-Vorpommern. Schwerin.

Neu, Claudia et al. 2012. Selbstversorgung im ländlichen Raum - eine empirische Analyse im Landkreis Adenau. Mönchengladbach (im Erscheinen).

Neu, Claudia; Nikolic, Ljubica 2013. Ankommen im Wandel. Rotenburg/Wümme (im Erscheinen).

Pauly, Walter 2001. "Die deutsche Staatsrechtslehre in der Zeit des Nationalsozialismus «, in Veröffentlichungen der Vereinigung der Deutschen Staatsrechtslehrer 60, S. 73-105.

Pries, Ludger. Hrsg. 1997. Transnationale Migration. Soziale Welt, Sonderband 12. BadenBaden: Nomos.

Pünder, Hermann 2008. „Gerechte Lastenverteilung zwischen den Generationen: Maßnahmen zur Reform des Staatsschuldenrechts und zur Vermeidung eines übermäßigen Defizits im Bundesstaat ", in Deutsches Verwaltungsblatt 123, 15, S. 946-956.

Reichel, Susanne 2009. Gleichwertigkeit der Lebensverhältnisse. Verfassungsauftrag und Raumordnungsrecht. München: C. H. Beck.

Runkel, Peter 2005. »Kommentierung des $\$ 1$ Raumordnungsgesetz «, in Raumordnungs- und Landesplanungsrecht des Bundes und der Länder, Band 2, hrsg. v. Bielenberg, Walter et al. Berlin: Erich Schmidt Verlag.

Schröder, Marlen 2007. Regionale Disparitäten - Herausforderungen für die Daseinsvorsorge. BA-Arbeit. Universität Rostock.

Schuppert, Gunnar F. 2000. Verwaltungswissenschaft. Baden-Baden: Nomos. 
Schuppert, Gunnar F. 2002. »Die Zukunft der Daseinsvorsorge in Europa: Zwischen Gemeinwohlbindung und Wettbewerb ", in Die Zukunft der kommunalen EVU im liberalisierten Energiemarkt, hrsg. v. Schwintowski, Hans-Peter, S. 11-40. Baden-Baden: Nomos.

Schütte, Christian 2006. Progressive Verwaltungsrechtswissenschaft auf konservativer Grundlage. Berlin: Duncker \& Humblot.

Vogel, Berthold 2007. Die Staatsbedürftigkeit der Gesellschaft. Hamburg: Hamburger Edition.

Vogel, Berthold 2009. Wohlstandkonflikte. Soziale Fragen, die aus der Mitte kommen. Hamburg: Hamburger Edition.

Wieland, Bernhard 2007. »Infrastruktur «, in Handbuch der Verkehrspolitik, hrsg. v. Schöller, Oliver; Canzler, Weert; Knie, Andreas, S. 376-404. Wiesbaden. VS Verlag für Sozialwissenschaften.

Zimmermann, Horst 2005. "Die Kraft des regionalen Unterschieds «, in Frankfurter Allgemeine Zeitung, 12. November 2005, S. 13. 
Zusammenfassung: Infrastrukturen sind nicht nur Vorleistungen für die Wirtschaft, bzw. Versorgungsleistungen für die Bevölkerung, sondern haben in entwickelten Wohlfahrtsstaaten sozial und territorial integrierende Funktionen durch ihre flächendeckende Bereitstellung, die allen Bürgern Zugang und Teilhabe an den gesellschaftlichen Entwicklungen ermöglicht. Wenn infolge eines demografischen Wandels Infrastruktur abgebaut wird, steht die soziale, wirtschaftliche und politische Kohäsion der Bundesrepublik zur Disposition. Die Antwort auf diese demografische Provokation liegt nicht im radikalen Rückzug der Infrastrukturen aus der Fläche, sondern in einer realistischen Ausdifferenzierung infrastruktureller Leistungserbringung.

Stichworte: demografischer Wandel, Daseinsvorsorge, Infrastruktur, Staatlichkeit, Teilhabe, Kohäsion

\section{Demographic change as infrastructural provocation}

Abstract: Infrastructure in developed welfare states has always been more than just inputs into the economy or services to the population - they also serve the function of social and territorial integration. Public services produce this integrating effect primarily because they are provided comprehensively, making it possible for citizens throughout the country to access and become involved in social developments. If demographic change leads to a widespread dismantling or shutdown of public infrastructure the social, economic, and political cohesion of the Federal Republic will be called into question. The appropriate response to this demographic "provocation « is not to radically reduce the area served by public infrastructure but to ensure the development of realistic and differentiated infrastructural services for the future.

Keywords: demographic change, infrastructure, public service, participation, cohesion

\section{AutorInnen}

Prof. Dr. Jens Kersten

Juristische Fakultät der Ludwig-Maximilians-Universität München

Lehrstuhl für Öffentliches Recht und Verwaltungswissenschaften

Ludwigstr. 28/Rgb.

80539 München

jens.kersten@jura.uni-muenchen.de

Prof. Dr. Claudia Neu

Professur für Allgemeine Soziologie

Hochschule Niederrhein

FB 05

Rheydter Str. 277

41065 Mönchengladbach

claudia.neu@hs-niederrhein.de

PD Dr. Berthold Vogel

Soziologisches Forschungsinstitut Göttingen (SOFI)

Friedländer Weg 31

37085 Göttingen

Berthold.Vogel@his-online.de 